\title{
RECURRENT WEIL-PETERSSON GEODESIC RAYS WITH NON-UNIQUELY ERGODIC ENDING LAMINATIONS
}

\author{
JEFFREY BROCK AND BABAK MODAMI
}

\begin{abstract}
We construct Weil-Petersson (WP) geodesic rays with minimal filling non-uniquely ergodic ending lamination which are recurrent to a compact subset of the moduli space of Riemann surfaces. This construction shows that an analogue of Masur's criterion for Teichmüller geodesics does not hold for WP geodesics.
\end{abstract}

\section{Contents}

1. Introduction 1

2. Background 3

2.1. The Curve Complex 3

2.2. The Weil-Petersson metric 12

3. Minimal non-uniquely ergodic laminations 14

4. Recurrence of geodesics 26

References

\section{INTRODUCTION}

The Weil-Petersson (WP) metric on Teichmüller space provides a negatively curved, Riemannian alternative to the more familiar Teichmüller metric, a Finsler metric whose global geometry is not negatively curved in any general sense. While negative curvature allows one to harness a broad range of techniques from hyperbolic geometry, difficulties in implementing these techniques arise from the fact that the WP metric is incomplete and that its sectional curvatures approach both 0 and $-\infty$ asymptotically near the completion. Nevertheless, it is useful to draw analogies between these metrics and instructive to determine which of these are robust or obtainable through methods in negative curvature.

As an example, in [BMM10] Brock, Masur and Minsky introduced a notion of an ending lamination for WP geodesic rays, an analogue of the vertical foliation associated a Teichmüller geodesic ray. They proved that the ending laminations parametrize the strong asymptote class of recurrent WP

Date: January 29, 2018.

2010 Mathematics Subject Classification. Primary 30F60, 32G15, Secondary 37D40.

The first author was partially supported by NSF grant DMS-1207572. 
geodesic rays. Recurrent rays are the rays whose projection to the moduli space recurs to a compact set infinitely often. Brock, Masur and Minsky BMM11 and Modami [Mod] initiated a systematic study of the behavior of Weil-Petersson geodesics in terms of their ending laminations and associated subsurface projection coefficients. Certain diophantine-type conditions for subsurface projection coefficients give strong control over the trajectories of the corresponding geodesics.

For example, criteria on these coefficients can be given to guarantee that geodesics projected to the moduli space stay in a compact part of the moduli space [BMM11, recur to a compact part of the moduli space, or diverge in the moduli space [Mod]. A simple scenario arises from bounding the subsurface coefficients associated to the ending lamination of all proper subsurfaces from above, akin to bounded-type irrational numbers, all of whose continued fraction coefficients are bounded. In this bounded type case the projection of the corresponding WP geodesic to the moduli space stays in a compact subset; we say the geodesic is co-bounded.

In this paper we prove

Theorem 1.1. There are Weil-Petersson geodesic rays in the Teichmüller space with minimal, filling, non-uniquely ergodic ending lamination whose projections to the moduli space are recurrent. Moreover, these rays are not contained in any compact subset of the moduli space.

The theorem sits in contrast with the following result of $\mathrm{H}$. Masur about Teichmüller geodesic rays with (minimal) non-uniquely ergodic vertical foliation. Note that a Teichmüller geodesic ray starting at a point $X$ in the Teichmüller space is determined by a unique holomorphic quadratic differential on $X$. For the description of Teichmüller geodesics in terms of holomorphic quadratic differentials and the associated vertical and horizontal measured foliations see e.g. Raf05.

Theorem 1.2. (Masur's criterion) [Mas92, Theorem 1.1] Suppose that the vertical foliation of a quadratic differential determining a Teichmüller geodesic ray is not uniquely ergodic. Then the Teichmüller geodesic is divergent in the moduli space.

Remark 1.3. Masur states and proves the theorem with the assumption that the vertical foliation is minimal. The same argument for each minimal component of the vertical foliation gives the Theorem 1.2 .

The contrapositive of the above theorem ensures that the vertical foliation (lamination) of a recurrent Teichmüller geodesic is uniquely ergodic. Comparing this fact and Theorem 1.1 exhibits an essential disparity between how the behavior of a Teichmüller geodesic is encoded in its vertical foliation (lamination) and how the behavior of a Weil-Petersson geodesic is encoded in its forward ending lamination. 
Remark 1.4. We remark that the methods here use explicit strong control over the family of geodesics in the Weil-Petersson metric with bounded nonannular combinatorics BMM11. We remark that in the low-complexity cases of the five-holed sphere and two-holed torus, the more complete control over Weil-Petersson geodesics obtained in [BM08] allows one to apply Theorem 2.13 to show that any Weil-Petersson geodesic with a filling ending lamination is recurrent. In this setting, then the mere existence of nonuniquely ergodic filling laminations shows the failure of Masur's Criterion in this setting. Here, we have chosen an explicit constructive approach that naturally generalizes to higher genus cases.

Acknowledgement: We would like to thank Chris Leininger and Kasra Rafi for very useful discussions. We would also like to thank Yair Minsky for continued support and encouragement.

\section{BACKGROUND}

In this paper we use the following notation:

Notation 2.1. Let $f, g: X \rightarrow \mathbb{R}^{\geq 0}$ be two function. Let $K \geq 1$ and $C \geq 0$ be two constants. We denote $f \asymp_{K, C} g$ if

$$
\frac{1}{K} g(x)-C \leq f(x) \leq K g(x)+C
$$

holds for every $x \in X$.

2.1. The Curve Complex. Let $S=S_{g, n}$ be a finite type, orientable surface with genus $g$ and $n$ punctures or boundary components. We define the complexity of the surface by $\xi(S):=3 g-3+n$. The curve complex of $S$ denoted by $\mathcal{C}(S)$ is a flag complex. When $\xi(S)>1$ : Each vertex in the complex is the isotopy class of an essential, simple closed curve. An essential curve is a curve which is not isotopic to a point, puncture or a boundary component of $S$. An edge corresponds to a pair of isotopy classes of simple closed curves with disjoint representatives on the surface. The curve complex is the flag complex obtained from the first skeleton i.e we have a $k$ dimensional simplex corresponding to any $k+1$ vertices with an edge between any pair of them. Assigning length one to each edge makes the first skeleton of the curve complex a metric graph. When $\xi(S)=1, S$ is a four-holed sphere or a one-holed torus. The definition of the curve complex is the same, except disjoint representative is replaced with intersection number 2 or 1 , respectively.

An essential subsurface of $S$ is a connected, closed, properly embedded subsurface $Y \subseteq S$, so that each boundary curve of $Y$ is either an essential simple closed curve of $S$ or a boundary curve of $S$, and $Y$ itself is not three-holed sphere. We frequently consider the inclusion of subcomplexes $\mathcal{C}(Y) \subseteq \mathcal{C}(S)$ induced by restriction. 
For an essential annular subsurface $Y$ with core curve $\alpha$, the curve complex has a slightly more involved definition, but a simple model: it is quasiisometric to $\mathbb{Z}$. Formally, let $\langle\alpha\rangle$ be the cyclic subgroup of $\pi_{1}(S)$ generated by $\alpha$ acting on the Poincaré disk $\mathbb{D}^{2}$ the universal cover of $S$. Let $\tilde{Y}=\mathbb{D}^{2} /\langle\alpha\rangle$ be the annular cover of $S$ to which $Y$ lifts homeomorphically. Let $\widehat{Y}=\mathbb{D}^{2} \cup \Omega_{\alpha} /\langle\alpha\rangle$ be the natural compactification of $\widetilde{Y}$, where $\Omega_{\alpha}$ is the complement of the fixed points of $\alpha$ acting on the circle at infinity of $\mathbb{D}^{2}$. Each vertex of $\mathcal{C}(Y)$ corresponds to the homotopy class of an arc connecting the two boundaries of $\widehat{Y}$ relative to the boundary. There is an edge between any two vertices corresponding to arcs with disjoint interior. We denote $\mathcal{C}(Y)$ by $\mathcal{C}(\alpha)$ as well. For more detail see $\S 2$ of [MM00].

We do not distinguish between the isotopy class of closed curve and any curve representing the class. A multi-curve is a collection of pairwise disjoint simple closed curves.

Masur-Minsky MM99 showed that the curve complex of a surface $S$ is $\delta$-hyperbolic where $\delta$ depends only on the topological type of the surface. Indeed, it has recently been shown that $\delta$ is universal, and can be taken to be the constant 17, HPW15] (see alos [Aou13]).

Notation 2.2. We say that curves $\alpha, \beta \in \mathcal{C}_{0}(S)$ overlap if $\alpha$ and $\beta$ cannot be realized by disjoint curves on $S$. If $\alpha$ and $\beta$ overlap we say that $\alpha \pitchfork \beta$ holds. A curve $\alpha$ overlaps a subsurface $Y$ if $\alpha$ can not be realized disjoint from $Y$; we denote it by $\alpha \pitchfork Y$. Multi-curves $\sigma$ and $\sigma^{\prime}$ overlap if some $\alpha \in \sigma$ and some $\alpha^{\prime} \in \sigma^{\prime}$ overlap. Similarly a multi-curve $\sigma$ and a subsurface $Y$ overlap if some $\alpha \in \sigma$ and $Y$ overlap.

Let $Y$ and $Z$ be essential subsurfaces. We say that $Y$ and $Z$ overlap if $\partial Z \pitchfork Y$ and $\partial Y \pitchfork Z$ hold.

Pants decompositions and markings: A pants decomposition $P$ is a multi-curve with maximal number of curves $\xi(S)$. A (partial) making $\mu$ consists of a pants deposition $P$ and $t_{\alpha}$ a diameter 1 subset of $\mathcal{C}_{0}(\alpha)$ for (some) all $\alpha \in P$. The subset of $\mathcal{C}_{0}(\alpha)$ can be represented by transversal curves to $\alpha$ on $S$. We call $P$ the base of the marking and denote it by $\operatorname{base}(\mu)$.

The pants graph of $S$ denoted by $P(S)$ is a graph with vertices the pants decompositions. An edge is between any two pants decompositions that differ by an elementary move. An elementary move on a pants decomposition $P$ fixes all the curves and replaces one curve $\alpha$ with a curve in $S \backslash\{P-\alpha\}$ whose intersection number with $\alpha$ is 1 if $S \backslash\{P-\alpha\}$ is a one-holed torus, and is 2 if $S \backslash\{P-\alpha\}$ is a four-holed sphere. Assigning length one to each edge we obtain a metric graph.

Laminations and foliations: Fix a complete hyperbolic metric on $S$. A geodesic lamination $\lambda$ is a closed subset of $S$ consisting of disjoint, complete, simple geodesics. Each one of the geodesics is called a leaf of $\lambda$. Let $\widetilde{S}=\mathbb{D}^{2}$ be the universal cover of $S$. Denote the circle at infinity of the Poincaré disk 
$\mathbb{D}^{2}$ by $\widetilde{S}_{\infty}$. Let $M_{\infty}(S)$ denote $\left(\widetilde{S}_{\infty} \times \widetilde{S}_{\infty} \backslash \Delta\right) / \sim$, where $\Delta$ is the diagonal and $\sim$ is the equivalence relation generated by $(x, y) \sim(y, x)$. Since the geodesics in $\mathbb{D}^{2}$ are parametrized by points of $M_{\infty}$ the preimage of a geodesic lamination determines a closed subset of $M_{\infty}(S)$ which is invariant under the action of $\pi_{1}(S)$. We denote the space of geodesic laminations on $S$ equipped with the Hausdorff topology of closed subsets of $M_{\infty}(S)$ by $\mathcal{G} \mathcal{L}(S)$. The space $\mathcal{G} \mathcal{L}(S)$ is a compact space. For more detail see $\S$ I.4 of [CEG06]. A transverse measure $m$ on $\lambda$ is a measure on the set of arcs on $S$ which is invariant under isotopies of $S$ preserving $\lambda$. The measure of an arc $a$ such that $a \subset \lambda$ or $a \cap \lambda=\emptyset$ is 0 and otherwise the measure of $a$ is positive. A pair of a geodesic lamination $\lambda$ and a transverse measure $m$ of $\lambda$ is a measured (geodesic) lamination, denoted by $\mathcal{L}=(\lambda, m)$. We say that $\lambda$ is the support of the measured lamination. We denote the space of measured laminations of $S$ equipped with the weak* topology by $\mathcal{M L}(S)$. The space of projective measured laminations $\mathcal{P} \mathcal{M L}(S)$ is the quotient of $\mathcal{M L}(S)$ with the natural action of $\mathbb{R}^{+}$rescaling the measures equipped with the quotient topology. For any $\mathcal{L} \in \mathcal{M L}(S)$, let $[\mathcal{L}]$ denote the projective class of $\mathcal{L}$.

A geodesic lamination $\lambda$ is minimal if every leaf of $\lambda$ is dense in $\lambda$. The geodesic lamination $\lambda$ fills the surface $S$ or is filling if $S \backslash \lambda$ is the union of topological disks and annuli with core curve isotopic to a boundary curve of $S$. Equivalently, if for any simple closed curve $\alpha$, and any transverse measure $m$ on $\lambda$, we have $i(\alpha,(\lambda, m))>0$. Here

$$
i: \mathcal{M L}(S) \times \mathcal{M L}(S) \rightarrow \mathbb{R}^{\geq 0}
$$

denotes the natural extension of the intersection number of curves to the space of measured geodesic laminations; see Bon01.

Given $[\mathcal{L}] \in \mathcal{P} \mathcal{M L}(S)$, let $|\mathcal{L}|$ be the support of $\mathcal{L}$. Then taking the quotient

$$
\mathcal{P} \mathcal{M L}(S) /|\cdot|
$$

of $\mathcal{P} \mathcal{M L}(S)$ by forgetting the measure, the ending lamination space

$$
\mathcal{E} \mathcal{L}(S) \subset \mathcal{P} \mathcal{M L}(S) / \mid \text {.| }
$$

is the image of projective measured laminations with minimal filling support equipped with the quotient topology of the topology of $\mathcal{P} \mathcal{M L}(S)$.

Recall that the curve complex of $S$ is a $\delta$-hyperbolic space. The following result of Klarriech describes the Gromov boundary of the curve complex.

Proposition 2.3. Kla There is a homeomorphism $\Phi$ from the Gromov boundary of $\mathcal{C}(S)$ equipped with its standard topology to $\mathcal{E} \mathcal{L}(S)$. Let $\left\{\alpha_{i}\right\}_{i=0}^{\infty}$ be a sequence of curves in $\mathcal{C}_{0}(S)$ that converges to a point $x$ in the Gromov boundary of $\mathcal{C}(S)$. Regarding each $\alpha_{i}$ as a projective measured lamination, any accumulation point of the sequence $\left\{\alpha_{i}\right\}_{i=0}^{\infty}$ in $\mathcal{P} \mathcal{M L}(S)$ is supported on $\Phi(x)$.

A singular foliation $\mathcal{F}$ on $S$ is a foliation of the complement of a finite set of points in $S$ called singular points. At a regular (not a singular) point $\mathcal{F}$ is 
locally modeled on an open set $U \subset \mathbb{C}$ containing the origin whose leaves are the horizontal coordinate lines. More precisely, there is a coordinate chart $x+i y$ such that the leaves of $\mathcal{F}$ are the trajectories given by $y=$ constant. At singular points the foliation is locally modeled on an open set $U \subset \mathbb{C}$ containing the origin whose leaves are the trajectories along which the real valued $1-$ form $\operatorname{lm}\left(\sqrt{z^{k} d z^{2}}\right)$ vanishes, where $k \in \mathbb{N}$. The singular point is mapped to the origin. A foliation is minimal if any half leaf of the foliation is dense in the surface.

A transverse measure on a singular foliation $\mathcal{F}$ is a measure on the collection of arcs in the surface transversal to $\mathcal{F}$ which is invariant under isotopies of the surface that preserve the foliation.

A pair consisting of a foliation and a transverse measure on the foliation is a measured foliation. Given a foliation $\mathcal{F}$, let $x+i y$ be a coordinate chart as above. Then $|d y|$ defines a transverse measure on $\mathcal{F}$.

We denote the space of measured foliations of the surface $S$ equipped with the weak* topology by $\mathcal{M F}(S)$. For more detail see exposé 5 of [FLP79].

There is a one to one correspondence between measured laminations and measured foliation up to Whitehead moves and isotopies of foliations on a surface [Lev83]. A lamination is minimal if and only if the corresponding foliation is minimal, see [Lev83, Theorem 2].

Subsurface coefficients: Let $Y \subseteq S$ be an essential non-annular subsurface. Masur and Minsky [MM99] define subsurface projection map

$$
\pi_{Y}: \mathcal{G L}(S) \rightarrow \mathcal{P C}_{0}(Y)
$$

that assigns to $\lambda \in \mathcal{G L}(S)$ the subset of $\mathcal{C}_{0}(S)$ denoted by $\pi_{Y}(\lambda)$ as follows: Fix a complete hyperbolic metric on $S$ and realize $\lambda$ and $\partial Y$ geodesically. If $\lambda$ does not intersect $Y$, then define $\pi_{Y}(\lambda)=\emptyset$. Now suppose that $\lambda$ intersects $Y$. Let $\lambda \cap Y$ be the intersection locus of $\lambda$ and the subsurface $Y$. Consider isotopy classes of arcs in $\lambda \cap Y$ with end points on $\partial Y$ or at cusps of the hyperbolic surface, where the end points of arcs are allowed to move in $\partial Y$. For any arc $a$ (up to isotopy) take the essential boundary curves of a regular neighborhood of $a \cup \partial Y$ in $Y$. The union of these curves where we select one arc from each isotopy class and the closed curves in $\lambda \cap Y$ is $\pi_{Y}(\lambda)$. Note that the diameter of $\pi_{Y}(\lambda)$ viewed as a subset of $\mathcal{C}(Y)$ is at most 2 .

Let $Y$ be an essential annular subsurface with core curve $\alpha$. Denote the natural compactification of the annular cover of $S$ to which $Y$ lifts homeomorphically by $\widehat{Y}$. Given a geodesic lamination $\lambda$, the projection of $\lambda$ to $Y$ is the set of component arcs of the lift of $\lambda$ to $\widehat{Y}$ which connect the two boundaries of $\widehat{Y}$. We denote the projection map by either $\pi_{Y}$ or $\pi_{\alpha}$. For more detail see [MM00, §2].

Note that since $\mathcal{C}_{0}(S) \subset \mathcal{G} \mathcal{L}(S)$, we have in particular the subsurface projection map

$$
\pi_{Y}: \mathcal{C}_{0}(S) \rightarrow \mathcal{P} \mathcal{C}_{0}(Y)
$$


Given a multi-curve $\sigma$ and an essential subsurface $Y$, the projection of $\sigma$ onto $Y$ is the union of the projections $\pi_{Y}(\alpha)$ where $\alpha \in \sigma$. For a partial marking $\mu$ if the subsurface $Y$ is not an annulus with core curve in base $(\mu)$, then $\pi_{Y}(\mu)=\pi_{Y}($ base $(\mu))$. If $Y$ is an annulus with core curve $\alpha \in \operatorname{base}(\mu)$, then $\pi_{Y}(\mu)$ is the set of transversal curves to $\alpha$ in $\mu$.

Let $\mu$ and $\mu^{\prime}$ be either partial markings or laminations. Let $Y \subseteq S$ be an essential subsurface. The $Y$ subsurface coefficient of $\mu$ and $\mu^{\prime}$ is defined by

$$
d_{Y}\left(\mu, \mu^{\prime}\right):=\min \left\{d_{Y}\left(\gamma, \gamma^{\prime}\right): \gamma \in \pi_{Y}(\mu), \gamma^{\prime} \in \pi_{Y}\left(\mu^{\prime}\right)\right\} .
$$

When $Y$ is an annular subsurface with core curve $\alpha$ we denote $d_{Y}(.,$.$) by$ $d_{\alpha}(.,$.$) as well.$

Lemma 2.1 of MM99 gives us the following bound on the subsurface coefficient of two curves in terms of their intersection number,

$$
d_{Y}(\alpha, \beta) \leq 2 i(\alpha, \beta)+1 .
$$

Let $\alpha, \beta \in \mathcal{C}_{0}(S)$ and $Y \subseteq S$ be an essential subsurface. If $d_{Y}(\alpha, \beta)>2$, then $\alpha \pitchfork \beta$ holds. To see this, first suppose that $Y$ is non-annular. Recall the surgery map which assigns to any arc in $Y$ with end points on $\partial Y$ the set of curves in the boundary of a regular neighborhood of $a \cup \partial Y$. This map from the arc complex of $Y$ to $\mathcal{P C}_{0}(Y)$ is 2-Lipschitz, see [MM00]. Let $a$ be an arc in $\alpha \cap Y$ and $b$ be an arc in $\beta \cap Y$ with end points in the boundary of $Y$. The assumption $d_{Y}(\alpha, \beta)>2$ and the fact that the surgery map is 2 -Lipschitz imply that $a$ and $b$ have arc complex distance at least 2 . Thus the arcs $a$ and $b$ intersect, and therefore the curves $\alpha$ and $\beta$ intersect. Now suppose that $Y$ is an annular subsurface. Then $d_{Y}(\alpha, \beta)>2$ implies that the interior of any two lifts of $\alpha$ and $\beta$ to the compactified annular cover $\widehat{Y}$ that go between two boundary components of $\widehat{Y}$ intersect. Therefore, $\alpha$ and $\beta$ intersect.

The following lemma is a consequence of [MM00, Lemma 2.3].

Lemma 2.4. Let $\mu$ denote a multi-curve, (partial) marking or lamination on a surface $S$. Then for any essential subsurface $Y \subseteq S$ we have

$$
\operatorname{diam}_{Y}(\mu) \leq 2 \text {. }
$$

When $Y$ is an annulus the sharp upper bound is 1.

The reason for the second part of the lemma is that any two lifts of two disjoint curves on the surface $S$ to the compactified annular cover corresponding to the annular subsurface $Y \subset S$ are disjoint.

Let $\alpha, \beta, \gamma \in \mathcal{C}_{0}(S)$. Farb, Lubotzky and Minsky [FLM01 defined the relative twist of the curves $\beta$ and $\gamma$ with respect to the curve $\alpha$ by

$$
\tau_{\alpha}(\beta, \gamma):=\left\{b . c: b \in \pi_{\alpha}(\beta), c \in \pi_{\alpha}(\gamma)\right\}
$$

where $b . c$ denotes the algebraic intersection number of the arcs $a$ and $b$. The arcs $b$ and $c$ are oriented so that they intersect the lift of $\alpha$ homotope to the core of $\widehat{Y}$ in the same direction. More precisely, let $\tilde{\alpha}$ be the lift of $\alpha$ 
homotopic to the core of $\widehat{Y}$ and fix an orientation for $\tilde{\alpha}$. Then $b$ and $c$ are oriented so that the tangents to $\tilde{\alpha}$ and $b$, and the tangents to $\tilde{\alpha}$ and $c$ at their intersection points determine the same orientation for the annulus $\tilde{Y}$. Note that The subset $\tau_{\alpha}(\beta, \gamma) \subset \mathbb{Z}$ has diameter 2 .

Given $\operatorname{arcs} b, c \in \mathcal{C}(\alpha)$, by the discussion in $\S 2.4$ of [MM00],

$$
d_{\alpha}(b, c)=|b . c|+1 \text {. }
$$

Let $\beta, \gamma \in \mathcal{C}_{0}(S)$. Since the diameter of $\tau_{\alpha}(\beta, \gamma)$ is at most 2 , by the above formula we have

$$
\left|d_{\alpha}(\beta, \gamma)-\right| x|| \leq 3
$$

for any $x \in \tau_{\alpha}(\beta, \gamma)$. Let $\gamma=\mathcal{D}_{\alpha}^{e}(\beta)$, where $\mathcal{D}_{\alpha}$ is the positive Dehn twist about $\alpha$ and $e$ is a positive integer. Formula (2) in [FLM01, §2] is

$$
\tau_{\alpha}(\beta, \gamma) \subset\{e, e+1\} .
$$

The following inequality proved by Behrstock Beh06 relates the subsurface coefficients of two subsurfaces that overlap.

Theorem 2.5. (Behrstock inequality) There is a constant $B_{0}>0$ so that given a curve system $\mu$ and subsurfaces $Y$ and $Z$ satisfying $Y \pitchfork Z$ we have

$$
\min \left\{d_{Y}(\partial Z, \mu), d_{Z}(\partial Y, \mu)\right\} \leq B_{0} .
$$

Remark 2.6. We note that Chris Leininger has observed that $B_{0}$ can be taken to be the universal constant 3 . However the specific value of $B_{0}$ does not play any role in our work.

Limits of laminations: Let $\mathcal{L}_{i}=\left(\lambda_{i}, m_{i}\right)(i \in \mathbb{N})$ be a sequence of measured laminations which converges to a measured lamination $\mathcal{L}=(\lambda, m)$ in the weak* topology. Suppose that after possibly passing to a subsequence, the laminations $\lambda_{i}$ converge to a lamination $\xi$ in the Hausdorff topology of $M_{\infty}(S)$. It is a standard fact that $\lambda \subseteq \xi$, see for example $\S$ I.4 of [CEG06].

Lemma 2.7. Suppose that a sequence of curves $\left\{\alpha_{i}\right\}_{i=0}^{\infty}$ converges to a lamination $\lambda$ in the Hausdorff topology of $M_{\infty}(S)$. Let $Y$ be an essential subsurface so that $\lambda$ intersects $Y$ essentially. Then for any geodesic lamination $\lambda^{\prime}$ that intersects $Y$ essentially, we have

$$
d_{Y}\left(\alpha_{i}, \lambda^{\prime}\right) \asymp_{1,4} d_{Y}\left(\lambda, \lambda^{\prime}\right),
$$

for all $i$ sufficiently large.

Proof. First suppose that $Y$ is an essential non-annular subsurface. Equip $S$ with a complete hyperbolic metric and realize $\partial Y$, the curves $\alpha_{i}$ and the lamination $\lambda$ geodesically in this metric. Let $b$ an arc in $\lambda \cap Y$ and $\delta>0$ be so that the $\delta$-neighborhood of $b \cup \partial Y$ is a regular neighborhood and at least one of the components of the boundary of the neighborhood is an essential curve in $Y$. Denote the neighborhood by $U$, see Figure 1 . Let $l$ be the geodesic in $\lambda$ so that $b \subset l$. Let $\tilde{l}$ be a lift of $l$ to the universal cover $\mathbb{D}^{2}$. The convergence of the curves $\alpha_{i}$ to $\lambda$ in the Hausdorff topology of $M_{\infty}(S)$ (see 


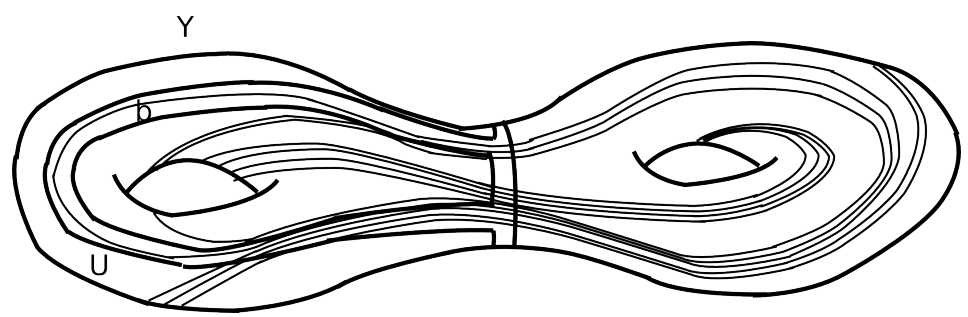

Figure 1. The $\delta$ neighborhood $U$ of an $\operatorname{arc} b$ in $\lambda \cap Y$ is a regular neighborhood in $Y$ with at least one essential boundary curve. For $i$ sufficiently large, the Hausdorff distance of $\alpha_{i}$ and $\lambda$ is less than $\delta$, so $\alpha_{i} \cap Y$ is contained in $U$.

Lemma I.4.1.11 in CEG06, §I.4]) guarantees that given $\delta^{\prime}<\delta$ and $L>0$, for all $i$ sufficiently large, there is a lift $\tilde{\alpha}_{i}$ of $\alpha_{i}$ to $\mathbb{D}^{2}$ so that $\tilde{\alpha}_{i}$ and $\tilde{l}$, $\delta^{\prime}$-fellow travel on an interval of length at least $L$. Then projecting $\tilde{\alpha}_{i}$ and $\tilde{l}$ to $S$ we can see that there is an $\operatorname{arc} a_{i}$ of $\alpha_{i} \cap Y$ such that the $\operatorname{arcs} b$ and $a_{i}$ are $\delta^{\prime}$ fellow travelers in $Y$. This implies that the regular neighborhood $U$ is also a regular neighborhood of $a_{i} \cup \partial Y$. By the definition of the subsurface projection the essential boundary curve of this neighborhood is a curve in $\pi_{Y}\left(\alpha_{i}\right)$.

By Lemma 2.4, $\pi_{Y}(\lambda)$ and $\pi_{Y}\left(\alpha_{i}\right)$ are subsets of $\mathcal{C}_{0}(Y)$ with diameter at most 2. Moreover as we saw in the previous paragraph $\pi_{Y}(\lambda) \cap \pi_{Y}\left(\alpha_{i}\right) \neq \emptyset$. Therefore

$$
\operatorname{diam}_{Y}\left(\pi_{Y}\left(\alpha_{i}\right) \cup \pi_{Y}(\lambda)\right) \leq 4 .
$$

Let $\beta$ be a curve in $\pi_{Y}\left(\lambda^{\prime}\right)$. Then by the above bound on the diameter we have

$$
\left|d_{Y}\left(\beta, \alpha_{i}\right)-d_{Y}(\beta, \lambda)\right| \leq 4
$$

This completes the proof of the lemma for non-annular subsurface $Y$.

Now suppose that $Y$ is an essential annular subsurface with core curve $\gamma$. Let $b$ be an arc in $\pi_{Y}(\lambda)$. We claim that, for all $i$ sufficiently large, there is an arc $a_{i}$ in $\pi_{Y}\left(\alpha_{i}\right)$ such that $a_{i}$ and $b$ have at most one intersection point in their interior. If $a_{i}$ and $b$ do not intersect we are done. Otherwise, after conjugation we may assume that the origin of $\mathbb{D}^{2}$ is a lift of an intersection point of $a_{i}$ and $b$. Moreover there are $\tilde{b}$ a lift of $b$ and $\tilde{a}_{i}$ a lift of $a_{i}$ to $\overline{\mathbb{D}^{2}}$ which pass through the origin, see Figure 2, As in Figure 2, there is a lower bound for the distance of $\tilde{b}$ and any other lift of $b$ to $\mathbb{D}^{2}$. Then choosing $\delta>0$ sufficiently small and $L>0$ large enough, any geodesic in $\mathbb{D}^{2}$ passing through the origin which $\delta$ fellow travels $\tilde{b}$ on an interval of length at least $L$ does not intersect any of the lifts of $b$ except $\tilde{b}$. The geodesic $\tilde{b}$ is a lift of a leaf of $\lambda$ to $\mathbb{D}^{2}$ and $\tilde{a}_{i}$ is a lift of $\alpha_{i}$ to $\mathbb{D}^{2}$. So the Hausdorff convergence of the curves $\alpha_{i}$ to $\lambda$ implies that given $\delta, L>0$, for $i$ sufficiently large $\tilde{a}_{i}$, $\delta$ fellow travels $\tilde{b}$ on an interval of length at least $L$. Therefore as we saw above $\tilde{a}_{i}$ intersects $\tilde{b}$ once at the origin and does not intersect any other lift 

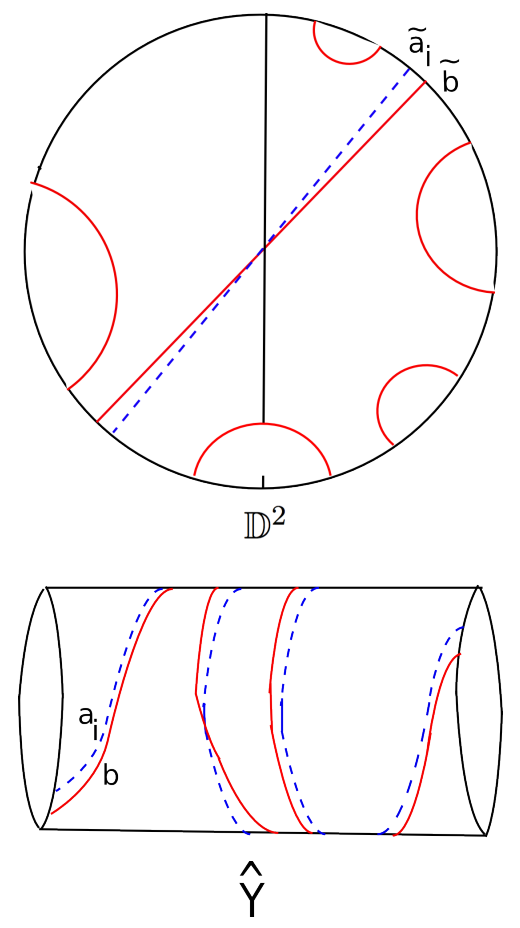

Figure 2. Bottom: The arc $b$ in $\pi_{Y}(\lambda)$ and $a_{i}$ in $\pi_{Y}\left(\alpha_{i}\right)$ in the compactified annular cover $\widehat{Y}$. Top: The lift $\tilde{b}$ of the arc $b$ and $\tilde{a}_{i}$ of $a_{i}$ to the universal cover $\mathbb{D}^{2}$ that pass through the origin and fellow travel for a long portion. As in the picture $\tilde{a}_{i}$ and $\tilde{b}$ intersect once, moreover $\tilde{a}_{i}$ does not intersect any other lift of $b$ to $\mathbb{D}^{2}$. Thus $b$ and $a_{i}$ in $\widehat{Y}$ intersect once.

of $b$. The number of times that the $\operatorname{arcs} a_{i}$ and $b$ intersect is equal to the number times that $\tilde{b}$ intersects all of the lifts of $a_{i}$ to $\mathbb{D}^{2}$. Which by the above discussion is at most 1 (see Figure 2). The proof of the claim is complete.

The fact that $a_{i}$ and $b$ intersect at most once implies that

$$
d_{Y}\left(\alpha_{i}, \lambda\right) \leq 2 .
$$

By Lemma 2.4, $\pi_{Y}\left(\alpha_{i}\right)$ and $\pi_{Y}(\lambda)$ are subsets of $\mathcal{C}_{0}(Y)$ with diameter at most 1 . Moreover as we saw above $\pi_{Y}\left(\alpha_{i}\right)$ and $\pi_{Y}(\lambda)$ have distance at most 2. Therefore

$$
\operatorname{diam}_{Y}\left(\pi_{Y}\left(\alpha_{i}\right) \cup \pi_{Y}(\lambda)\right) \leq 4 .
$$

Let $\beta$ be a curve in $\pi_{Y}\left(\lambda^{\prime}\right)$. Then by the above bound on the diameter we have

$$
\left|d_{Y}\left(\beta, \alpha_{i}\right)-d_{Y}(\beta, \lambda)\right| \leq 4
$$

This completes the proof of the lemma for annular subsurface $Y$.

Hierarchy paths and the distance formula: Hierarchy paths introduced by Masur and Minsky [MM00], comprise quasi-geodesics in the pants 
and marking graphs of a surface with constants depending only on the topological type of the surface. Hierarchy paths have properties encoded in their end points and the associated subsurface coefficients. For a list of these properties see [BMM11, §2] and [Mod, §2]. Here we only state a key feature of hierarchy paths which is the no backtracking property. For other properties we provide a reference wherever we use them.

Theorem 2.8. There exists a constant $M_{2}>0$ depending only on the topological type of the surface $S$ with the following property. Let $\rho:[m, n] \rightarrow$ $P(S)$ be a hierarchy path. Let $i, j, k, l \in[m, n]$ with $i \leq j \leq k \leq l$. For any subsurface $Y \subseteq S$ we have

$$
d_{Y}(\rho(i), \rho(l))+2 M_{2} \geq d_{Y}(\rho(j), \rho(k)) .
$$

The following theorem is a straightforward consequence of the Bounded Geodesic Image Theorem [MM00, Theorem 3.1].

Theorem 2.9. Given $k \geq 1$ and $c \geq 0$, there is a $G \geq 0$ with the following property. Let $\left\{\gamma_{i}\right\}_{i=0}^{\infty}$ be a sequence of curves in $\mathcal{C}_{0}(S)$ which form a 1-Lipschitz, $(k, c)$-quasi-geodesic. Let $Y \subsetneq S$ be an essential subsurface so that $\gamma_{i} \pitchfork Y$ holds for all $i \geq 0$, then

$$
\operatorname{diam}_{Y}\left(\left\{\pi_{Y}\left(\gamma_{i}\right)\right\}_{i=0}^{\infty}\right) \leq G .
$$

Here $\operatorname{diam}_{Y}($.$) is the diameter of the given subset of \mathcal{C}(Y)$.

Using the hierarchical machinery Masur and Minsky provide the following quasi-distance formula in the pants graph of a surface [MM00, Theorem 6.12]. Given $A>M_{1}$ ( $M_{1}$ is a constant depending on the topological type of $S$ ) there are constants $K \geq 1$ and $C \geq 0$ such that

$$
d\left(\mu, \mu^{\prime}\right) \asymp_{K, C} \sum_{\substack{Y \subseteq S \\ \text { non-annular }}}\left\{d_{Y}\left(\mu, \mu^{\prime}\right)\right\}_{A} .
$$

Here the cut-off function $\{.\}_{A}: \mathbb{R} \rightarrow \mathbb{R}^{\geq 0}$ is defined by

$$
\{a\}_{A}= \begin{cases}a & \text { if } a \geq A \\ 0 & \text { if } a<A\end{cases}
$$

Bounded combinatorics: A pair of laminations or partial markings $\left(\mu, \mu^{\prime}\right)$ has non-annular $R$-bounded combinatorics if

$$
d_{Y}\left(\mu, \mu^{\prime}\right) \leq R
$$

for every proper, essential, non-annular subsurface $Y \subsetneq S$.

The following result about stability of hierarchy paths with non-annular bounded combinatorics in the pants graph is an important ingredient in the proof that bounded combinatorics of end invariants of a WP geodesic guarantees co-boundedness of the geodesic and vice versa (see [BMM11]). We need this theorem in our study of the behavior of WP geodesics in 4 . 
Theorem 2.10. BMM11] Given $R>0$ there is a quantifier function

$$
d_{R}: \mathbb{R}^{\geq 1} \times \mathbb{R}^{\geq 0} \rightarrow \mathbb{R}^{\geq 0}
$$

so that a hierarchy path $\rho$ with end points with non-annular $R$-bounded combinatorics is $d_{R}$-stable in the pants graph. That is, any $(K, C)-q u a s i-$ geodesic with end points on $|\rho|$ stays in the $d_{R}(K, C)$ neighborhood of $|\rho|$. Here $|\rho|$ is the union of the pants decompositions of $\rho$.

2.2. The Weil-Petersson metric. In this section we assemble properties of the Weil-Petersson metric we will need. For an introduction to the synthetic geometry of the Weil-Petersson metric see [Wol10].

The Weil-Petersson metric on the Teichmüller space Teich $(S)$ is a Riemannian metric with negative sectional curvatures. It is incomplete, but is geodesically convex: any two points are joined by a unique geodesic that lies in the interior. Its metric completion $\overline{\text { Teich }(S)}$ is a CAT $(0)$ space. See $\S$ II.3.4 of [BH99] for an introduction to CAT(0) space. By the work of H. Masur [Mas76] the completion of the Teichmüller space with the Weil-Petersson metric is naturally identified with the augmented Teichmüller space obtained by adjoining nodal surfaces as limits. The completion is stratified by the data of simple closed curves on $S$ that are pinched: each stratum $\mathcal{S}(\sigma)$ is a copy of the Teichmüller space of the surface $S \backslash \sigma$, where $\sigma$ is a multicurve. Masur also gave an expansion of the metric near the completion showing that the inclusion $\mathcal{S}(\sigma) \hookrightarrow$ Teich $(S)$ is an isometry and $\mathcal{S}(\sigma)$ is totally geodesic.

S. Yamada observed that a stronger form of Masur's expansion should hold near the completion guaranteeing that the Weil-Petersson metric is asymptotic to a metric product of strata to higher order, and work of Daskalopolous-Wentworth [DW03] gave the appropriate metric expansion. Their expansion showed that these completion strata have the non-refraction property: For any $X, Y \in \overline{\operatorname{Teich}(S)}$, the interior of the unique geodesic connecting $X$ and $Y$ lies in the smallest stratum that contains $X$ and $Y$. See Wol03 for stronger form of the asymptotic expansion of the WP metric. The Weil-Petersson metric is invariant under the action of the mapping class group of the surface $\operatorname{Mod}(S)$ and descends under the natural orbifold cover to a metric on the moduli space of Riemann surfaces $\mathcal{M}(S)$. The completion descends to a metric on the familiar Deligne-Mumford compactification of $\mathcal{M}(S)$.

Length-functions: Let $X \in \operatorname{Teich}(S)$. Let $\alpha$ be a closed curve on $S$. We denote by $\ell_{\alpha}(X)$ the length of the geodesic representative of $\alpha$ in its free homotopy class on $S$. The length-function has a natural extension to the space of measured laminations, [Bon01]. For $\mathcal{L} \in \mathcal{M L}(S)$, we denote the length of $\mathcal{L}$ by $\ell_{\mathcal{L}}(X)$.

Significant from the point of view of the Weil-Petersson geometry is the result of Wolpert, Wol10, that each length-function is a strictly convex function along any WP geodesic. 
Quasi-isometric model: Let $S$ be a surface with negative Euler characteristic. There is a constant $L_{S}$ (Bers constant) depending only on the topological type of $S$ such that any complete hyperbolic metric on $S$ has a pants decomposition (Bers pants decomposition) with the property that the length of any curve in the pants decomposition is at most $L_{S}$, see Bus10. $\S 5]$. Let $X \in \overline{\operatorname{Teich}(S)}$. Suppose that $X \in \mathcal{S}(\sigma)$. A Bers pants decomposition of $X$, denoted by $Q(X)$, is the union of Bers pants decompositions of the connected components of $S \backslash \sigma$ and $\sigma$. A Bers marking of $X$, denoted by $\mu(X)$, is a partial marking obtained from a Bers pants decomposition $Q$ of $X$ by adding a transversal curve with minimal length for each $\alpha \in Q-\sigma$. The following result of Jeffrey Brock provides a quasi-isometric model for the Weil-Petersson metric.

Theorem 2.11. (Quasi-isometric model) Bro03. There are constants $K_{\mathrm{WP}} \geq$ 1 and $C_{\mathrm{WP}} \geq 0$ depending only on the topological type of $S$ with the following property. The map $Q: \operatorname{Teich}(S) \rightarrow P(S)$, assigning to each $X$ a Bers pants decomposition of $X$ is a $\left(K_{\mathrm{WP}}, C_{\mathrm{WP}}\right)$-quasi-isometry.

Ending laminations: Let $r:[0, a) \rightarrow$ Teich $(S)$ be a WP geodesic ray. Any limit in the weak* topology of an infinite sequence of distinct Bers curves at $r\left(t_{n}\right)$ where $t_{n} \rightarrow a$ is an ending measured lamination of $r$. A pinching curve $\alpha$ along $r$ is a curve so that $\ell_{\alpha}(r(t)) \rightarrow 0$ as $t \rightarrow a$. In [BMM10] Brock, Masur and Minksy showed that the union of the supports of ending measured laminations and pinching curves of $r$ is a geodesic lamination. We call this lamination the ending lamination of $r$.

Let $g:(a, b) \rightarrow$ Teich $(S)$ be a WP geodesic, where $(a, b)$ is an open interval containing 0 . If the forward trajectory $\left.g\right|_{[0, b)}$ can be extended to $b$ so that $g(b) \in \overline{\text { Teich }(S)}$ we define the forward end invariant of $g$ to be a Bers partial marking of $g(b)$. If not let the forward end invariant of $g$ be the lamination of $\left.g\right|_{[0, b)}$ we defined above. We denote the forward end invariant by $\nu^{+}=\nu^{+}(g)$. Similarly, consider the backward trajectory $\left.g\right|_{(a, 0]}$ and define the backward end invariant of $g, \nu^{-}=\nu^{-}(g)$.

From $\S 8$ of [Mod] we have the following result:

Lemma 2.12. (Infinite rays) Let $\nu$ be a minimal filling lamination. There is an infinite WP geodesic ray $r$ with forward ending lamination $\nu$.

The following strengthened version of Wolpert's Geodesic Limit Theorem (see [Wol03] and [BMM11]) proved in $\S 4$ of [Mod] provides a limiting picture for a sequence of bounded length WP geodesic segments in the Teichmüller space.

Theorem 2.13. (Geodesic limits) Given $T>0$. Let $\zeta_{n}:[0, T] \rightarrow \overline{\operatorname{Teich}(S)}$ be a sequence of WP geodesic segments parametrized by arc-length. After possibly passing to a subsequence there is a partition $0=t_{0}<\ldots .<t_{k+1}=T$ of $[0, T]$, possibly empty multi-curves $\sigma_{0}, \ldots, \sigma_{k+1}$ and a multi-curve $\hat{\tau} \equiv$ 
$\sigma_{i} \cap \sigma_{i+1}$ for $i=0,1, \ldots, k$ and a piece-wise geodesic

$$
\hat{\zeta}:[0, T] \rightarrow \overline{\operatorname{Teich}(S)},
$$

with the following properties

(1) $\hat{\zeta}\left(\left(t_{i}, t_{i+1}\right)\right) \subset \mathcal{S}(\hat{\tau})$ for $i=0, \ldots, k$,

(2) $\hat{\zeta}\left(t_{i}\right) \in \mathcal{S}\left(\sigma_{i}\right)$ for $i=0, \ldots, k+1$,

Given a multi-curve $\sigma$ denote by $\operatorname{tw}(\sigma)$ the subgroup of $\operatorname{Mod}(S)$ generated by positive Dehn twists about the curves in $\sigma$. There are elements of the mapping class group $\psi_{n}$ for each $n \in \mathbb{N}$, and $\mathcal{T}_{i, n} \in \operatorname{tw}\left(\sigma_{i}-\hat{\tau}\right)$ for $i=1, \ldots, k$ and $n \in \mathbb{N}$ so that

(3) $\psi_{n}\left(\zeta_{n}(t)\right) \rightarrow \hat{\zeta}(t)$ as $n \rightarrow \infty$ for all $t \in\left[0, t_{1}\right]$. Let $\varphi_{i, n}=\mathcal{T}_{i, n} \circ \ldots \circ \mathcal{T}_{1, n} \circ \psi_{n}$ for $i=1, \ldots, k$ and each $n \in \mathbb{N}$. Then $\varphi_{i, n}\left(\zeta_{n}(t)\right) \rightarrow \hat{\zeta}(t)$ for any $t \in$ $\left[t_{i}, t_{i+1}\right]$ as $n \rightarrow \infty$.

Remark 2.14. The central difference between the above version and original versions lies in the assertion that we have one (possibly empty) multicurve $\hat{\tau}$ rather than several multi-curves $\tau_{i}=\sigma_{i} \cap \sigma_{i+1}, i=0,1, . ., k$, allowed in Wolpert's Geodesic Limit Theorem. In particular, in part (1) the geodesic segments $\hat{\zeta}\left(\left(t_{i}, t_{i+1}\right)\right)$ lie in one stratum $\mathcal{S}(\hat{\tau})$ rather than several strata $\mathcal{S}\left(\tau_{i}\right)$.

\section{Minimal nOn-Uniquely ERgodic laminations}

A (measurable) geodesic lamination $\lambda$ is non-uniquely ergodic if there are non-proportional measures supported on $\lambda$. More precisely, $\lambda$ is nonuniquely ergodic if there exist transverse measures $m$ and $m^{\prime}$ supported on $\lambda$ and curves $\alpha$ and $\beta$ such that

$$
\frac{m(\alpha)}{m^{\prime}(\alpha)} \neq \frac{m(\beta)}{m^{\prime}(\beta)} .
$$

Gabai [Gab09, §9] gave a recipe to construct minimal filling non-uniquely ergodic geodesic laminations on any surface $S$ with $\xi(S)>1$. In fact, Gabai outlined the construction of minimal filling laminations and measures supported on each one of them with distinct projective classes Gab09, Theorem 9.1]. Leininger-Lenzhen-Rafi [LLR, §3-5] gave a detailed construction of minimal filling non-uniquely ergodic laminations on the surface $S_{0,5}$. Moreover, they studied the set of measures supported on the lamination and their projective classes.

We first recall the construction of $[\mathrm{LLR}]$. Let $\left\{e_{i}\right\}_{i=1}^{\infty}$ be a sequence of positive integers. Let $\rho: S_{0,5} \rightarrow S_{0,5}$ be the order-five homeomorphism of $S_{0,5}$ realized as the rotation by angle $\frac{4 \pi}{5}$ in Figure 3 . Let $\mathcal{D}=\mathcal{D}_{\hat{\gamma}_{2}}$ be the positive Dehn twist about the curve $\hat{\gamma}_{2}$. Let $f_{i}=\mathcal{D}^{e_{i}} \circ \rho$, for $i \geq 1$. Define the sequence of curves $\hat{\gamma}_{i}=f_{1} \circ f_{2} \circ \ldots \circ f_{i}\left(\hat{\gamma}_{0}\right)$, for $i \geq 1$. The curves $\hat{\gamma}_{0}, \ldots, \hat{\gamma}_{5}$ are shown in Figure 3 .

Proposition 3.1. There are constants $E>0, k \geq 1, c \geq 0$, and $K \geq$ $1, C \geq 0$ with the following properties. Suppose that $\left\{e_{i}\right\}_{i=1}^{\infty}$ is a sequence 

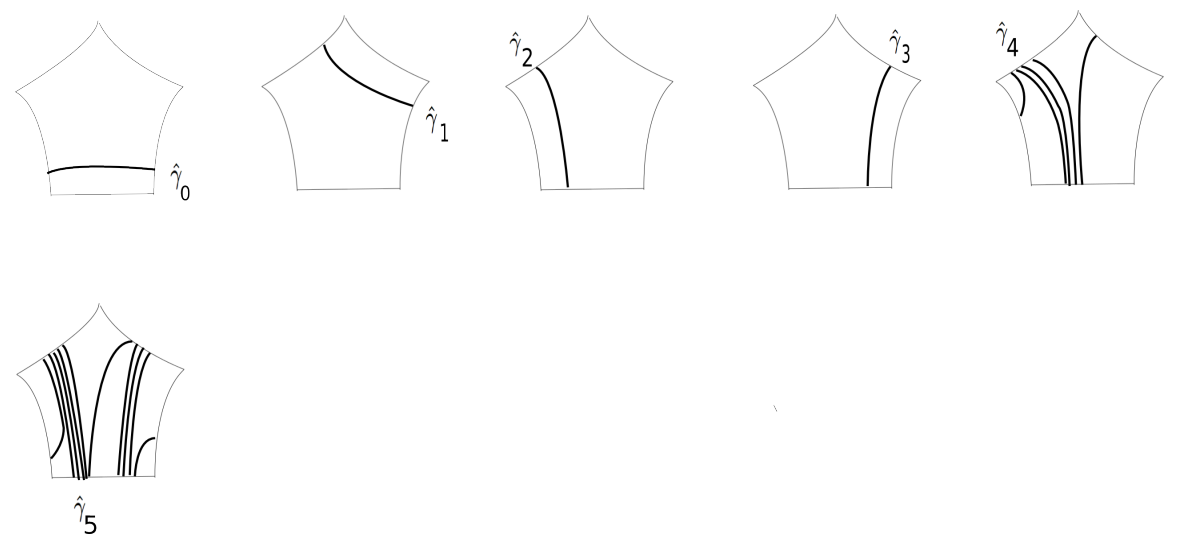

FiguRE 3. The double of each pentagon in the picture is a five-times punctured 2-sphere. Let the curves $\hat{\gamma}_{0}, \hat{\gamma}_{1}, \ldots, \hat{\gamma}_{5}$ are shown in the picture. Any other six consecutive curves in the sequence after applying an appropriate element of $\operatorname{Mod}\left(S_{0,5}\right)$ are the same as the above six curves, where the last two curves have different number of parallel strands from $\hat{\gamma}_{4}$ and $\hat{\gamma}_{5}$, respectively.

of integers satisfying $e_{i}>E$ for all $i \geq 1$. Let $\left\{\hat{\gamma}_{i}\right\}_{i=0}^{\infty}$ be the sequence of curves described. Then:

(1) For any $i \geq 0$ and $j \geq i+2, \hat{\gamma}_{j} \pitchfork \hat{\gamma}_{i}$ holds.

(2) For any $i \geq 0$ and $j \geq i+4$ the curves $\hat{\gamma}_{i}$ and $\hat{\gamma}_{j}$ fill the surface $S_{0,5}$.

(3) The sequence of curves $\left\{\hat{\gamma}_{i}\right\}_{i=0}^{\infty}$ is a 1-Lipschitz, $(k, c)$-quasi-geodesic in $\mathcal{C}_{0}\left(S_{0,5}\right)$.

(4) $d_{\hat{\gamma}_{i}}\left(\hat{\gamma}_{j}, \hat{\gamma}_{j^{\prime}}\right) \asymp_{K, C} e_{i-1}$ for any $j \geq i+2$ and $j^{\prime} \leq i-2$.

Proof. We start by proving the following lemma.

Lemma 3.2. Let $i \geq 1$. For any $j \geq i+2$ and $j^{\prime} \leq i-2$,

$$
\hat{\gamma}_{i} \pitchfork \hat{\gamma}_{j} \quad \text { and } \quad \gamma_{i} \pitchfork \hat{\gamma}_{j^{\prime}}
$$

hold. Furthermore, the subsurface coefficient bound

$$
d_{\hat{\gamma}_{i}}\left(\hat{\gamma}_{j}, \hat{\gamma}_{j^{\prime}}\right) \geq e_{i-1}-C
$$

holds.

Here $C=2 B_{0}+7$ and $B_{0}$ is the constant from Theorem 2.5 (Behrstock Inequality).

Proof. Set the constant

$$
E=C+B_{0}+G_{0}+2
$$

where $G_{0}$ is the constant from Theorem 2.9 for a geodesic in the curve complex of $S_{0,5}$.

We prove (3.1) and (3.2) simultaneously by induction on $j-j^{\prime}$. The proof of the base of the induction breaks into the following cases: 
Case $j-j^{\prime}=4, j-i=2$ and $i-j^{\prime}=2$ : Applying $\left(f_{1} \circ \ldots \circ f_{i-2}\right)^{-1}$ to the curves $\hat{\gamma}_{j^{\prime}}, \hat{\gamma}_{i}$ and $\hat{\gamma}_{j}$, we obtain the curves

$$
\hat{\gamma}_{0}, \hat{\gamma}_{2}=f_{i-1} \circ f_{i}\left(\hat{\gamma}_{0}\right) \text { and } f_{i-1} \circ \ldots \circ f_{i+2}\left(\hat{\gamma}_{0}\right) \text {, }
$$

respectively. The curve $f_{i-1} \circ \ldots \circ f_{i+2}\left(\hat{\gamma}_{0}\right)$ is the same as $\hat{\gamma}_{4}$ with a different number of parallel strands, see Figure 3 . Since $\hat{\gamma}_{2} \pitchfork \hat{\gamma}_{0}$ and $\hat{\gamma}_{2} \pitchfork f_{i-1} \circ \ldots \circ$ $f_{i+2}\left(\hat{\gamma}_{0}\right)$ hold, it follows that $\hat{\gamma}_{i} \pitchfork \hat{\gamma}_{i-2}$ and $\hat{\gamma}_{i} \pitchfork \hat{\gamma}_{i+2}$ hold. This is (3.1).

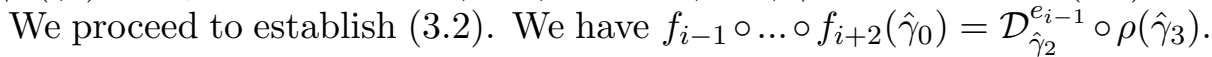
Then by the formula $(2.3)$ for the relative twists we have

$$
\tau_{\hat{\gamma}_{2}}\left(f_{i-1} \circ \ldots \circ f_{i+2}\left(\hat{\gamma}_{0}\right), \rho\left(\hat{\gamma}_{3}\right)\right) \subset\left\{e_{i-1}, e_{i-1}+1\right\} .
$$

Then $(2.2)$ implies that

$$
d_{\hat{\gamma}_{2}}\left(f_{i-1} \circ \ldots \circ f_{i+2}\left(\hat{\gamma}_{0}\right), \rho\left(\hat{\gamma}_{3}\right)\right) \geq e_{i-1}-3 .
$$

Furthermore, the curves $\rho\left(\hat{\gamma}_{3}\right)$ and $\hat{\gamma}_{0}$ are disjoint and both intersect $\hat{\gamma}_{2}$, thus

$$
d_{\hat{\gamma}_{2}}\left(\hat{\gamma}_{0}, \rho\left(\hat{\gamma}_{3}\right)\right) \leq 1 \text {. }
$$

Combining the above two subsurface coefficient bounds by the triangle inequality and using the fact that $\operatorname{diam}_{\hat{\gamma}_{2}}\left(\rho\left(\hat{\gamma}_{3}\right)\right) \leq 1$, we have

$$
d_{\hat{\gamma}_{2}}\left(\hat{\gamma}_{0}, f_{i-1} \circ \ldots \circ f_{i+2}\left(\hat{\gamma}_{i}\right)\right) \geq e_{i-1}-5 .
$$

Applying $f_{1} \circ \ldots \circ f_{i-2}$ to the subsurface coefficient above and using the fact that $C>5$, we obtain

$$
d_{\hat{\gamma}_{i}}\left(\hat{\gamma}_{i-2}, \hat{\gamma}_{i+2}\right) \geq e_{i-1}-C .
$$

This is the subsurface coefficient bound $(3.2)$.

Case $j-j^{\prime}=5, i-j^{\prime}=2$ and $j-i=3$ : Applying $\left(f_{1} \circ \ldots \circ f_{i-2}\right)^{-1}$ to the curves $\hat{\gamma}_{j^{\prime}}, \hat{\gamma}_{i}$ and $\hat{\gamma}_{j}$, we obtain the curves

$$
\hat{\gamma}_{0}, \hat{\gamma}_{2}=f_{i-1} \circ f_{i}\left(\hat{\gamma}_{0}\right) \text { and } f_{i-1} \circ \ldots \circ f_{i+3}\left(\hat{\gamma}_{0}\right) \text {, }
$$

respectively. The curve $f_{i-1} \circ \ldots \circ f_{i+3}\left(\hat{\gamma}_{0}\right)$ is the same as $\hat{\gamma}_{5}$ with a different number of parallel strands, see Figure 3. Since $\hat{\gamma}_{0} \pitchfork \hat{\gamma}_{2}$ and $\hat{\gamma}_{2} \pitchfork f_{i-1} \circ \ldots \circ$ $f_{i+3}\left(\hat{\gamma}_{0}\right)$ hold, (3.1) holds.

We have $f_{i-1} \circ \ldots \circ f_{i+3}\left(\hat{\gamma}_{i}\right)=\mathcal{D}_{\hat{\gamma}_{2}}^{e_{i-1}} \circ \rho\left(f_{i} \circ \ldots \circ f_{i+3}\left(\hat{\gamma}_{i}\right)\right)$. Then by 2.3),

$$
\tau_{\hat{\gamma}_{2}}\left(f_{i-1} \circ \ldots \circ f_{i+3}\left(\hat{\gamma}_{i}\right), \rho \circ f_{i} \circ \ldots \circ f_{i+3}\left(\hat{\gamma}_{i}\right)\right) \subset\left\{e_{i-1}, e_{i-1}+1\right\} .
$$

So (2.2) implies that

$$
d_{\hat{\gamma}_{2}}\left(f_{i-1} \circ \ldots \circ f_{i+3}\left(\hat{\gamma}_{i}\right), \rho \circ f_{i} \circ \ldots \circ f_{i+3}\left(\hat{\gamma}_{i}\right)\right) \geq e_{i-1}-3 .
$$

Furthermore, because $\rho\left(\hat{\gamma}_{3}\right)$ is a curve intersecting $\hat{\gamma}_{2}$ and disjoint from both $\hat{\gamma}_{0}$ and $\rho \circ f_{i} \circ \ldots \circ f_{i+3}\left(\hat{\gamma}_{i}\right)$ (to see this, note that $f_{i} \circ \ldots \circ f_{i+3}\left(\hat{\gamma}_{i}\right)$ is $\hat{\gamma}_{4}$ with different number of parallel strands), we have

$$
d_{\hat{\gamma}_{2}}\left(\hat{\gamma}_{0}, \rho \circ f_{i} \circ \ldots \circ f_{i+3}\left(\hat{\gamma}_{i}\right)\right) \leq 2 .
$$


Combining the above two subsurface coefficient bounds by the triangle inequality and using the fact that $\operatorname{diam}_{\hat{\gamma}_{2}}\left(\rho \circ f_{i} \circ \ldots \circ f_{i+3}\left(\hat{\gamma}_{i}\right)\right) \leq 1$, we have

$$
d_{\hat{\gamma}_{2}}\left(\hat{\gamma}_{0}, f_{i-1} \circ \ldots \circ f_{i+3}\left(\hat{\gamma}_{i}\right)\right) \geq e_{i-1}-6 .
$$

Now applying $f_{1} \circ \ldots \circ f_{i-2}$ to the above subsurface coefficient and using the fact that $C>6$, we get

$$
d_{\hat{\gamma}_{i}}\left(\hat{\gamma}_{i-2}, \hat{\gamma}_{i+3}\right) \geq e_{i-1}-C .
$$

This is the subsurface coefficient bound (3.2).

Case $j-j^{\prime}=5, i-j^{\prime}=3$ and $j-i=2$ : Applying $\left(f_{1} \circ \ldots \circ f_{i-3}\right)^{-1}$ to the curves $\hat{\gamma}_{j^{\prime}}, \hat{\gamma}_{i}$ and $\hat{\gamma}_{j}$, we obtain the curves

$$
\hat{\gamma}_{0}, \hat{\gamma}_{3}=f_{i-2} \circ f_{i-1} \circ f_{i}\left(\hat{\gamma}_{0}\right) \text { and } f_{i-2} \circ \ldots \circ f_{i+2}\left(\hat{\gamma}_{0}\right),
$$

see Figure 3. The statement about the intersection of curves (3.1) holds since $\hat{\gamma}_{3} \pitchfork \hat{\gamma}_{0}$ and $\hat{\gamma}_{3} \pitchfork f_{i-2} \circ \ldots \circ f_{i+2}\left(\hat{\gamma}_{0}\right)$.

By the triangle inequality

$$
\begin{aligned}
d_{\hat{\gamma}_{3}}\left(\hat{\gamma}_{0}, f_{i-2} \circ \ldots \circ f_{i+2}\left(\hat{\gamma}_{0}\right)\right) & \geq d_{\hat{\gamma}_{3}}\left(\hat{\gamma}_{1}, f_{i-2} \circ \ldots \circ f_{i+2}\left(\hat{\gamma}_{0}\right)\right) \\
& -d_{\hat{\gamma}_{3}}\left(\hat{\gamma}_{0}, \hat{\gamma}_{1}\right)-\operatorname{diam}_{\hat{\gamma}_{3}}\left(\hat{\gamma}_{1}\right) .
\end{aligned}
$$

First we find a lower bound for the first term on the right-hand side of (3.4). Note that $f_{i-2}\left(\hat{\gamma}_{0}\right)=\hat{\gamma}_{1}$ and $f_{i-2}\left(\hat{\gamma}_{2}\right)=\hat{\gamma}_{3}$. Thus applying $\left(f_{i-2}\right)^{-1}$ to this term, we obtain

$$
d_{\hat{\gamma}_{2}}\left(\hat{\gamma}_{0}, f_{i-1} \circ \ldots \circ f_{i+2}\left(\hat{\gamma}_{0}\right)\right) \text {. }
$$

This subsurface coefficient by $(3.3)$ is bounded below by $e_{i-1}-5$.

The two curves $\hat{\gamma}_{0}$ and $\hat{\gamma}_{1}$ are disjoint and intersect $\hat{\gamma}_{3}$. So the second term on the right-hand side of $(3.4)$ is bounded by 1 .

These bounds for the two terms on the right-hand side of the inequality (3.4) and the fact that $\operatorname{diam}_{\hat{\gamma}_{3}}\left(\hat{\gamma}_{1}\right) \leq 1$ (Lemma 2.4) give us

$$
d_{\hat{\gamma}_{3}}\left(\hat{\gamma}_{0}, f_{i-2} \circ \ldots \circ f_{i+2}\left(\hat{\gamma}_{0}\right)\right) \geq e_{i-1}-7>e_{i-1}-C .
$$

Applying $f_{1} \circ \ldots \circ f_{i-1}$ to the subsurface coefficient on the left-hand side of the above inequality, we obtain the bound (3.2).

We proved that (3.1) and $(3.2)$ hold for $j-j^{\prime} \leq 5$. In what follows we assume that (3.1) and (3.2) hold when $j-j^{\prime} \leq n$, where $n \geq 6$, and prove that (3.1) and (3.2) hold for $j-j^{\prime}=n+1$

If $j-i=2$ or 3 , applying $\left(f_{1} \circ \ldots \circ f_{i}\right)^{-1}$ to $\hat{\gamma}_{i}$ and $\hat{\gamma}_{j}$ we obtain $\hat{\gamma}_{0}$ and $\hat{\gamma}_{j-i}$, respectively. Then since $\hat{\gamma}_{0} \pitchfork \hat{\gamma}_{j-i}$ (see Figure 3 ), we have $\hat{\gamma}_{i} \pitchfork \hat{\gamma}_{j}$. If $j-i=4$ or 5 , then since $(j-2)-i \geq 2$ and $j-(j-2)=2$, by the hypothesis of the induction

$$
d_{\hat{\gamma}_{j-2}}\left(\hat{\gamma}_{i}, \hat{\gamma}_{j}\right) \geq E>2 .
$$

This bound implies that $\hat{\gamma}_{i} \pitchfork \hat{\gamma}_{j}$ holds. 
Now suppose that $j-i \geq 6$. Then we have $(j-2)-i \geq 2$. Thus by the induction hypothesis $\hat{\gamma}_{j-2} \pitchfork \hat{\gamma}_{i}$ holds. Moreover, $\hat{\gamma}_{j} \pitchfork \hat{\gamma}_{j+2}$. So we may write the following triangle inequality

$$
\begin{aligned}
d_{\hat{\gamma}_{j-2}}\left(\hat{\gamma}_{i}, \hat{\gamma}_{j}\right) & \geq d_{\hat{\gamma}_{j-2}}\left(\hat{\gamma}_{j-4}, \hat{\gamma}_{j}\right)-d_{\hat{\gamma}_{j-2}}\left(\hat{\gamma}_{j-4}, \hat{\gamma}_{i}\right)-\operatorname{diam}_{\hat{\gamma}_{j-2}}\left(\hat{\gamma}_{j-4}\right) \\
& \geq E-C-B_{0}-1>2 .
\end{aligned}
$$

To get the second inequality in (3.5), first, by the assumption of the induction, we have

$$
d_{\hat{\gamma}_{j-2}}\left(\hat{\gamma}_{j-4}, \hat{\gamma}_{j}\right) \geq e_{j-3}-C \geq E-C,
$$

This gives a lower bound for the first term on the right-hand side of the first inequality of (3.5). Second, since $(j-4)-i \geq 2$ by the assumption of the induction we have

$$
d_{\hat{\gamma}_{j-4}}\left(\hat{\gamma}_{i}, \hat{\gamma}_{j-2}\right) \geq e_{j-5}-C>E-C>B_{0}
$$

where the last inequality holds because $E>C+B_{0}$. Then Behrstock inequality (Theorem 2.5) implies that

$$
d_{\hat{\gamma}_{j-2}}\left(\hat{\gamma}_{j-4}, \hat{\gamma}_{i}\right) \leq B_{0} .
$$

This is the upper bound for the second term on the right-hand side of the first inequality of (3.5). Finally the last term by Lemma 2.4 is at most 1.

The lower bound (3.5) guarantees that $\hat{\gamma}_{i} \pitchfork \hat{\gamma}_{j}$ holds. The proof of that $\hat{\gamma}_{j^{\prime}} \pitchfork \hat{\gamma}_{i}$ holds for each $j^{\prime} \leq i-2$ is similar. The proof of (3.1) is complete.

We proceed to establish (3.2). Let $j, j^{\prime}$ be so that $j^{\prime} \leq i-2$ and $j \geq i+2$. By (3.1) we may write the following triangle inequality

$$
\begin{aligned}
d_{\hat{\gamma}_{i}}\left(\hat{\gamma}_{j^{\prime}}, \hat{\gamma}_{j}\right) & \geq d_{\hat{\gamma}_{i}}\left(\hat{\gamma}_{i-2}, \hat{\gamma}_{i+2}\right)-d_{\hat{\gamma}_{i}}\left(\hat{\gamma}_{i-2}, \hat{\gamma}_{j^{\prime}}\right)-d_{\hat{\gamma}_{i}}\left(\hat{\gamma}_{i+2}, \hat{\gamma}_{j}\right) \\
& -\operatorname{diam}_{\hat{\gamma}_{i}}\left(\hat{\gamma}_{i-2}\right)-\operatorname{diam}_{\hat{\gamma}_{i}}\left(\hat{\gamma}_{i+2}\right) .
\end{aligned}
$$

We have that $(i-2)-j^{\prime}<i-j^{\prime}<j-j^{\prime}$ and $j-(i+2)<j-i<j-j^{\prime}$. Thus by the assumption of the induction, the fact that $e_{i}>E$ and the choice of $E$ we have that

$$
\begin{aligned}
d_{\hat{\gamma}_{i-2}}\left(\hat{\gamma}_{i}, \hat{\gamma}_{j^{\prime}}\right) & \geq E-C>B_{0}, \text { and } \\
d_{\hat{\gamma}_{i+2}}\left(\hat{\gamma}_{i}, \hat{\gamma}_{j}\right) & \geq E-C>B_{0},
\end{aligned}
$$

The first lower bound above and the Behrstock inequality imply that the second term on the right-hand side of $(3.6)$ is bounded above by $B_{0}$. Similarly the second bound above and the Behrstock inequality imply that the third term on the right-hand side of $(3.6)$ is bounded above by $B_{0}$. Moreover, by Lemma 2.4 the third and fourth terms on the right-hand side of (3.6) are less than or equal to 1 . So we obtain

$$
\begin{aligned}
d_{\hat{\gamma}_{i}}\left(\hat{\gamma}_{j^{\prime}}, \hat{\gamma}_{j}\right) & \geq d_{\gamma_{i}}\left(\hat{\gamma}_{i-2}, \hat{\gamma}_{i+2}\right)-2 B_{0}-2 \\
& \geq e_{i-1}-C .
\end{aligned}
$$

The proof of $(3.2)$ is complete. 
We proceed to prove the proposition. Part (1) is the statement about intersection of curves (3.1) we proved in Lemma 3.2. Note that (3.2) gives the lower bound in part (4). Part (3) is Lemma 3.2 of [LLR]. Part (4) follows from parts (1), (3) and Theorem 2.9 (Bounded Geodesics Image Theorem).

Now we prove part (2) of the proposition. The proof is by induction on $j-i$ and is essentially the one given in Lemma 3.2 of [LLR]. Note that here we do not assume any upper bound for the value of $j-i$.

In the rest of the proof denote the surafce $S_{0,5}$ by $S$. Suppose that $j-i=$ 4. Applying $\left(f_{1} \circ \ldots \circ f_{i}\right)^{-1}$ to the curves $\hat{\gamma}_{i}$ and $\hat{\gamma}_{j}$ we obtain the curves $\hat{\gamma}_{0}$ and $\hat{\gamma}_{4}$ in Figure 3, respectively, which fill $S$. Thus $\hat{\gamma}_{i}$ and $\hat{\gamma}_{j}$ fill $S$.

Suppose that part (2) is true for all $j-i \leq n$, where $n \geq 5$. Let $j-i=$ $n+1$. To get a contradiction suppose that curves $\hat{\gamma}_{i}$ and $\hat{\gamma}_{j}$ do not fill the surface. Then $d_{S}\left(\hat{\gamma}_{i}, \hat{\gamma}_{j}\right) \leq 2$. On the other hand, by the assumption of the induction the curves $\hat{\gamma}_{i}$ and $\hat{\gamma}_{j-1}$ fill $S$, so $d_{S}\left(\hat{\gamma}_{i}, \hat{\gamma}_{j-1}\right) \geq 3$. Moreover, by the construction of the sequence of curves $\hat{\gamma}_{j}$ and $\hat{\gamma}_{j-1}$ are disjoint, so $d_{S}\left(\hat{\gamma}_{j}, \hat{\gamma}_{j-1}\right)=1$. Thus by the triangle inequality $d_{S}\left(\hat{\gamma}_{i}, \hat{\gamma}_{j}\right) \geq 2$. The two bounds we established for $d_{S}\left(\hat{\gamma}_{i}, \hat{\gamma}_{j}\right)$ imply that

$$
d_{S}\left(\hat{\gamma}_{i}, \hat{\gamma}_{j}\right)=2
$$

Since $j-i \geq 5$ we may choose an index

$$
h \text { so that } i<h<h+1<j, j-h-1 \geq 2 \text { and } h-i \geq 2 .
$$

Then by (3.1) the curves $\hat{\gamma}_{i}$ and $\hat{\gamma}_{j}$ intersect $\hat{\gamma}_{h}$ and $\hat{\gamma}_{h+1}$. Moreover, by the bound (3.2), the fact that $e_{i}>E$ and the choice of $E$ we have that

$$
\begin{aligned}
d_{\hat{\gamma}_{h}}\left(\hat{\gamma}_{i}, \hat{\gamma}_{j}\right) & \geq E-C>G_{0}, \text { and } \\
d_{\hat{\gamma}_{h+1}}\left(\hat{\gamma}_{i}, \hat{\gamma}_{j}\right) & \geq E-C>G_{0}
\end{aligned}
$$

where $G_{0}$ is the constant from Theorem 2.9 for a geodesic in $\mathcal{C}(S)$.

As we saw above $d_{S}\left(\hat{\gamma}_{j}, \hat{\gamma}_{i}\right)=2$, so the geodesic in $\mathcal{C}(S)$ connecting $\hat{\gamma}_{i}$ and $\hat{\gamma}_{j}$ contains three curves $\hat{\gamma}_{i}, \gamma^{\prime}$ and $\hat{\gamma}_{j}$. We have that the curve $\gamma^{\prime}$ is disjoint from $\hat{\gamma}_{h}$. For otherwise, the curves $\gamma_{i}, \gamma^{\prime}, \gamma_{j}$ which form a geodesic in $\mathcal{C}(S)$ intersect $\hat{\gamma}_{h}$. Then Theorem 2.9 (Bounded Geodesic Image) implies that $d_{\hat{\gamma}_{h}}\left(\hat{\gamma}_{i}, \hat{\gamma}_{j}\right) \leq G_{0}$. But this contradicts the first lower bound above. Similarly using the second lower bound above we may show that $\gamma^{\prime}$ and $\hat{\gamma}_{h+1}$ are disjoint. The curves $\hat{\gamma}_{h}$ and $\hat{\gamma}_{h+1}$ consist a pants decomposition on $S$. Thus the only curves disjoint from both $\hat{\gamma}_{h}$ and $\hat{\gamma}_{h+1}$ are themselves. So $\gamma^{\prime}$ is either $\hat{\gamma}_{h}$ or $\hat{\gamma}_{h+1}$. As we mentioned above the curves $\hat{\gamma}_{h}$ and $\hat{\gamma}_{h+1}$ intersect the curves $\hat{\gamma}_{i}$ and $\hat{\gamma}_{j}$. So $\gamma^{\prime}$ intersects both $\hat{\gamma}_{i}$ and $\hat{\gamma}_{j}$. On the other hand, since $\hat{\gamma}_{i}, \gamma^{\prime}$ and $\hat{\gamma}_{j}$ are consecutive curves on a geodesic in $\mathcal{C}(S)$, $\gamma^{\prime}$ is disjoint from both $\hat{\gamma}_{i}$ and $\hat{\gamma}_{j}$. This contradiction shows that in fact $\hat{\gamma}_{i}$ and $\hat{\gamma}_{j}$ fill $S$ and completes the proof of part (2) by induction.

Let the sequence of integers $\left\{e_{i}\right\}_{i=1}^{\infty}$ with $e_{i}>E$, and the sequence of curves $\left\{\hat{\gamma}_{i}\right\}_{i=0}^{\infty}$ be as in Proposition 3.1. Part (3) of Proposition 3.1 and 
hyperbolicity of the curve complex imply that the sequence of curves $\left\{\hat{\gamma}_{i}\right\}_{i=0}^{\infty}$ converges to a point in the Gromov boundary of the curve complex. By Proposition 2.3 this point determines a projective measured lamination $[\mathcal{E}]$ with minimal filling support $\hat{\nu}$ on $S_{0,5}$.

Proposition 3.3. Let the marking $\hat{\mu}$ and the geodesic lamination $\hat{\nu}$ be as above. We have

(1) There exist $K \geq 1$ and $C \geq 0$ so that $d_{\hat{\gamma}_{i}}(\hat{\mu}, \hat{\nu}) \asymp_{K, C} e_{i-1}$.

Furthermore, suppose that for some $a>2$ we have $e_{i+1} \geq a e_{i}$ for each $i \geq 1$. Then

(2) The geodesic lamination $\hat{\nu}$ is minimal, filling and non-uniquely ergodic.

Part (1) follows from Proposition 3.1 (4) and Theorem 2.9 (Bounded Geodesics Image Theorem). Part (2) is Theorem 1.1 of [LLR]. Note that the growth of powers $e_{i+1} \geq a e_{i}(a>2)$ is required to guarantee the nonunique ergodicity of the lamination $\hat{\nu}$.

The utility of the construction of Leininger-Lenzhen-Rafi lies in its control on subsurface coefficients; see Proposition 3.3 (1), Theorem 3.4 and Theorem 3.5. These are conditions similar to arithmetic conditions for coefficients of the continued fraction expansion of irrational numbers relevant to the coding of geodesics on the modular surface which is $\mathcal{M}\left(S_{1,1}\right)$ as well; see [Ser85]. Though Gabai's construction produces a minimal filling nonuniquely ergodic lamination on any surface $S$ with $\xi(S)>1$, it provides no a priori control on subsurface coefficients.

Theorem 3.4. There is a constant $\hat{R}>0$ such that for any proper, essential, non-annular subsurface $Y \subsetneq S$ we have $d_{Y}(\hat{\mu}, \hat{\nu}) \leq \hat{R}$. In other words, the pair $(\hat{\mu}, \hat{\nu})$ has non-annular $\hat{R}$-bounded combinatorics.

Proof. By Proposition 3.1 (3), $\left\{\hat{\gamma}_{i}\right\}_{i=0}^{\infty}$ is a 1-Lipschitz, $(k, c)$-quasi-geodesic in $\mathcal{C}\left(S_{0,5}\right)$. Let $G$ be the corresponding constant from Theorem 2.9 (Bounded Geodesic Image Theorem). Let $Y \subseteq S_{0,5}$ be an essential non-annular subsurface. First note that $Y$ is a four-holed sphere.

If $\hat{\gamma}_{i} \pitchfork Y$ holds for all $i \geq 0$, then the Bounded Geodesic Image Theorem guarantees that

$$
\operatorname{diam}_{Y}\left(\left\{\hat{\gamma}_{i}\right\}_{i=0}^{\infty}\right) \leq G .
$$

The lamination $\hat{\nu}$ is filling, so $\pi_{Y}(\hat{\nu}) \neq \emptyset$. Now we claim that

$$
d_{Y}(\hat{\mu}, \hat{\nu}) \leq G+6 .
$$

To see this, let $\hat{\gamma}_{j_{n}}$ be a convergent subsequence of $\left\{\hat{\gamma}_{j}\right\}_{j=i+2}^{\infty}$ in the $\mathcal{P} \mathcal{M L}(S)$ topology. By Proposition 2.3 the support of the limit of $\hat{\gamma}_{j_{n}}$ is $\hat{\nu}$. After possibly passing to a further subsequence we may assume that $\hat{\gamma}_{j_{n}}$ is also convergent in the Hausdorff topology of $M_{\infty}(S)$. Denote the limit lamination in the Hausdorff topology by $\xi$. Then $\hat{\nu} \subseteq \xi$ (see e.g. CEG06]). By the bound $\operatorname{diam}_{Y}\left(\left\{\hat{\gamma}_{i}\right\}_{i=0}^{\infty}\right) \leq G$ we established above, we have that $d_{Y}\left(\hat{\gamma}_{0}, \hat{\gamma}_{j_{n}}\right) \leq$ 
$G$. Then since $\hat{\gamma}_{j_{n}} \rightarrow \xi$ in the Hausdorff topology as $n \rightarrow \infty$, by Lemma 2.7 , we obtain

$$
d_{Y}\left(\xi, \hat{\gamma}_{0}\right) \leq G+4 \text {. }
$$

Furthermore we have that $\hat{\nu} \subseteq \xi$ and $\hat{\gamma}_{0} \subset \hat{\mu}$. Then $\operatorname{since} \operatorname{diam}_{Y}(\xi) \leq 2$ (by Lemma 2.4), the difference of the subsurface projection distance in (3.7) and the one above is most at 2. Which gives us (3.7).

Now suppose that for some integer $i \geq 0, \hat{\gamma}_{i} \pitchfork Y$ does not hold. Then since $Y$ is a four-holed sphere inside $S_{0,5}$ we have that $\partial Y=\hat{\gamma}_{i}$.

Let $j^{\prime} \leq i-2$. By part (1) of Proposition 3.1. $\hat{\gamma}_{i} \pitchfork \hat{\gamma}_{j^{\prime}}$. Then since $\partial Y=\hat{\gamma}_{i}$, we conclude that $\hat{\gamma}_{j^{\prime}} \pitchfork Y$ holds. Thus Bounded Geodesic Image Theorem guarantees that

$$
\operatorname{diam}_{Y}\left(\left\{\hat{\gamma}_{j^{\prime}}\right\}_{j^{\prime}=0}^{i-2}\right) \leq G
$$

The above bound and the fact that $\hat{\mu}$ contains $\hat{\gamma}_{0}$ give us the bound

$$
d_{Y}\left(\hat{\mu}, \hat{\gamma}_{i-2}\right) \leq G \text {. }
$$

Let $j \geq i+2$. By part (1) of Proposition 3.1. $\hat{\gamma}_{i} \pitchfork \hat{\gamma}_{j}$ holds. Then similarly to above we obtain that

$$
\operatorname{diam}_{Y}\left(\left\{\hat{\gamma}_{j}\right\}_{j=i+2}^{\infty}\right) \leq G .
$$

Then similar to the proof of (3.7) we may obtain

$$
d_{Y}\left(\hat{\nu}, \hat{\gamma}_{i+2}\right) \leq G+6 .
$$

By Proposition 3.1 (4) $\hat{\gamma}_{i-2} \pitchfork \hat{\gamma}_{i}$. So $\hat{\gamma}_{i-2} \pitchfork Y$ holds, because $\hat{\gamma}_{i}=\partial Y$. Similarly $\hat{\gamma}_{i+2} \pitchfork Y$ holds. So $d_{Y}\left(\hat{\gamma}_{i-2}, \hat{\gamma}_{i+2}\right)$ is defined. We claim that

$$
d_{Y}\left(\hat{\gamma}_{i-2}, \hat{\gamma}_{i+2}\right)=1 \text {. }
$$

To see this, let $g$ be the element of $\operatorname{Mod}(S)$ given by the composition $g=$ $f_{1} \circ \ldots \circ f_{i-2}$. Applying $g^{-1}$ to the subsurface coefficient in 3.10 we get

$$
d_{g^{-1}(Y)}\left(g^{-1}\left(\hat{\gamma}_{i-2}\right), g^{-1}\left(\hat{\gamma}_{i+2}\right)\right) .
$$

Thus, to obtain the desired equality, it suffices to show that the above subsurface coefficient is equal to 1 . The curves

$$
g^{-1}\left(\hat{\gamma}_{i-2}\right), \ldots, g^{-1}\left(\hat{\gamma}_{i+2}\right)
$$

are the curves $\hat{\gamma}_{0}, \ldots, \hat{\gamma}_{4}$ in Figure 3 , respectively, except that the twist of the curve $g^{-1}\left(\hat{\gamma}_{i+2}\right)$ about $g^{-1}\left(\hat{\gamma}_{i}\right)=\hat{\gamma}_{2}$ is $e_{i-1}$ rather than $e_{1}$.

Since $\partial Y=\hat{\gamma}_{i}$, the subsurface $g^{-1}(Y)$ is the four holed sphere with boundary $\hat{\gamma}_{2}$, see Figure 4 . We have that $g^{-1}\left(\hat{\gamma}_{i-2}\right)=\hat{\gamma}_{0}$. Furthermore, the curve $g^{-1}\left(\hat{\gamma}_{i+2}\right)$ is the curve $\hat{\gamma}_{4}$ in Figure 3 , except that the twist of the curve $g^{-1}\left(\hat{\gamma}_{i+2}\right)$ about $\hat{\gamma}_{2}$ is $e_{i-1}$ rather than $e_{1}$. The projection of the curves $g^{-1}\left(\hat{\gamma}_{i-2}\right)$ and $g^{-1}\left(\hat{\gamma}_{i+2}\right)$ to the subsurface $g^{-1}(Y)$ are shown in Figure 4. The $\mathcal{C}\left(g^{-1}(Y)\right)$-distance of these two curves is 1 , because these are two curves with (minimal) intersection number 2 on the four-holed sphere $g^{-1}(Y)$, yielding the desired equality. 


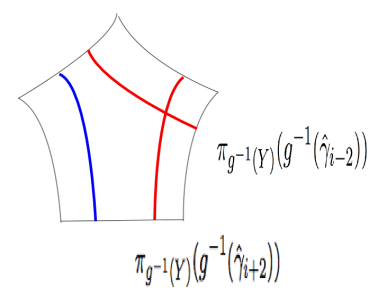

Figure 4. The subsurface $g^{-1}(Y) \subset S_{0,5}$ is the fourholed sphere with boundary $\partial g^{-1}(Y)=\hat{\gamma}_{2}$. The curves $\pi_{g^{-1}(Y)}\left(g^{-1}\left(\hat{\gamma}_{i-2}\right)\right)$ and $\pi_{g^{-1}(Y)}\left(g^{-1}\left(\hat{\gamma}_{i+2}\right)\right)$ are shown in the figure.

Note that $\hat{\mu}$ is a marking and $\hat{\nu}$ fills the surface. By the triangle inequality and the bounds $(3.8),(3.9)$ and $(3.10)$ we have

$$
\begin{aligned}
d_{Y}(\hat{\mu}, \hat{\nu}) & \leq d_{Y}\left(\hat{\mu}, \hat{\gamma}_{i-2}\right)+d_{Y}\left(\hat{\gamma}_{i-2}, \hat{\gamma}_{i+2}\right)+d_{Y}\left(\hat{\gamma}_{i+2}, \hat{\nu}\right) \\
& +\operatorname{diam}_{Y}\left(\hat{\gamma}_{i-2}\right)+\operatorname{diam}_{Y}\left(\hat{\gamma}_{i+2}\right) \\
& \leq 2 G+6+1+4 .
\end{aligned}
$$

We conclude that the $Y$ subsurface coefficient of $\hat{\mu}$ and $\hat{\nu}$ is bounded above by $\hat{R}:=2 G+11$, as was desired.

Theorem 3.5. There is a constant $R^{\prime}>0$, so that $d_{\beta}(\hat{\mu}, \hat{\nu}) \leq R^{\prime}$ for any curve $\beta$ which is not in the sequence $\left\{\hat{\gamma}_{i}\right\}_{i=0}^{\infty}$.

Proof. By Proposition 3.1 (3), $\left\{\hat{\gamma}_{i}\right\}_{i=0}^{\infty}$ is a 1-Lipschitz, $(k, c)$-quasi-geodesic in $\mathcal{C}\left(S_{0,5}\right)$. Let $G$ be the corresponding constant from Theorem 2.9 (Bounded Geodesic Image Theorem).

If $\beta$ intersects all of the curves in the sequence. Then similar to (3.7) in the proof of Theorem 3.4 we may obtain that

$$
d_{\beta}(\hat{\mu}, \hat{\nu}) \leq G+5 .
$$

Now suppose that for some integer $i>0$ the curve $\beta$ is disjoint from $\hat{\gamma}_{i}$. By Proposition 3.1(2) for every $j \geq i+4$, the curves $\hat{\gamma}_{j}$ and $\hat{\gamma}_{i}$ fill $S_{0,5}$. So we may deduce that $\beta \pitchfork \hat{\gamma}_{j}$ holds. Then Theorem 2.9 guarantees that

$$
d_{\beta}\left(\hat{\mu}, \hat{\gamma}_{i-4}\right) \leq G \text {. }
$$

Similarly for every $j^{\prime} \leq i-4, \beta \pitchfork \hat{\gamma}_{j^{\prime}}$ holds. Then

$$
\operatorname{diam}_{\beta}\left(\left\{\gamma_{j^{\prime}}\right\}_{j^{\prime}=0}^{i-4}\right) .
$$

by the Bounded Geodesic Image Theorem. Then similarly to (3.7) we may obtain

$$
d_{\beta}\left(\hat{\nu}, \hat{\gamma}_{i+4}\right) \leq G+5
$$

Let $g=f_{1} \circ \ldots \circ f_{i}$. Applying $g^{-1}$ to the curves $\hat{\gamma}_{i}, \ldots, \hat{\gamma}_{i+4}$, we obtain the curves $\hat{\gamma}_{0}, \ldots, \hat{\gamma}_{4}$ in Figure 3 , respectively. The difference is that $g^{-1}\left(\hat{\gamma}_{i+4}\right)$ has $e_{i+1}$ twists. The only curve disjoint from $\hat{\gamma}_{0}$ and $\hat{\gamma}_{2}$ is $\hat{\gamma}_{1}$. Therefore, 
the only curve disjoint from $\hat{\gamma}_{i}$ and $\hat{\gamma}_{i+2}$ is $\hat{\gamma}_{i+1}$. The curve $\beta$ is not in the sequence $\left\{\hat{\gamma}_{i}\right\}_{i=0}^{\infty}$, in particular $\beta \neq \hat{\gamma}_{i+1}$. Moreover $\beta$ is disjoint from $\hat{\gamma}_{i}$. Thus $\beta \pitchfork \hat{\gamma}_{i+2}$ holds. Furthermore, the curves $\hat{\gamma}_{0}$ and $g^{-1}\left(\hat{\gamma}_{i+4}\right)$ fill $S$ $\left(g^{-1}\left(\hat{\gamma}_{i+4}\right)\right.$ is $\hat{\gamma}_{4}$ with a different number of parallel strands). Thus $\hat{\gamma}_{i}$ and $\hat{\gamma}_{i+4}$ fill $S$. Then since $\beta$ is disjoint from $\hat{\gamma}_{i}, \beta \pitchfork \hat{\gamma}_{i+4}$ holds. We showed that $\beta \pitchfork \hat{\gamma}_{i+2}$ and $\beta \pitchfork \hat{\gamma}_{i+4}$, therefore $d_{\beta}\left(\hat{\gamma}_{i+2}, \hat{\gamma}_{i+4}\right)$ is defined. Now since $i\left(\hat{\gamma}_{i+2}, \hat{\gamma}_{i+4}\right)=2$, by (2.1) we obtain the bound

$$
d_{\beta}\left(\hat{\gamma}_{i+2}, \hat{\gamma}_{i+4}\right) \leq 5 \text {. }
$$

Similarly, we may obtain the bound

$$
d_{\beta}\left(\hat{\gamma}_{i-2}, \hat{\gamma}_{i-4}\right) \leq 5 \text {. }
$$

Let $g=f_{1} \circ \ldots \circ f_{i-2}$. Applying $g^{-1}$ to the curves $\hat{\gamma}_{i-2}, \ldots, \hat{\gamma}_{i+2}$ we obtain the first five curves in Figure 3 , with the difference that the last curve has $e_{i-1}$ twists. Let $Y \subset S_{0,5}$ be the the four-holed sphere with boundary curve $\hat{\gamma}_{i}$. Then $\beta \in \mathcal{C}_{0}(Y)$. The curves $\pi_{g^{-1}(Y)}\left(g^{-1}\left(\hat{\gamma}_{i-2}\right)\right)$ and $\pi_{g^{-1}(Y)}\left(g^{-1}\left(\hat{\gamma}_{i+2}\right)\right)$ are shown in Figure 4. These two curves intersect twice. Thus by (2.1) we have

$$
d_{\beta}\left(\hat{\gamma}_{i-2}, \hat{\gamma}_{i+2}\right) \leq 5
$$

The bounds (3.11), (3.12), 3.13), (3.14) and (3.15) for the $\beta$ subsurface coefficients combined by the triangle inequality give us the bound $R^{\prime}:=$ $2 G+29$.

We proceed to construct minimal, filling, non-uniquely ergodic laminations on any surface $S_{g, 0}$ of genus $g \geq 2$ with control on the subsurface coefficients of the laminations. We construct the laminations by an appropriate lift of the lamination we described on $S_{0,5}$ using 2-dimensional orbifolds and their orbifold covers. Here, we replace each puncture with a marked point on the surface.

Let $\mathcal{S}_{0,5}$ be the 2 sphere equipped with an orbifold structure with five orbifold points of order 2 at the 5 marked points of $S_{0,5}$. Let $\mathcal{S}_{0,6}$ be the 2 sphere with an orbifold structure with orbifold points of order 2 at the marked points of $S_{0,6}$. Let $\mathcal{S}_{g, 0}(g \geq 2)$ be $S_{g, 0}$ equipped with an orbifold structure with no orbifold point (i.e. a manifold structure). Let

$$
f: \mathcal{S}_{0,6} \rightarrow \mathcal{S}_{0,5} \quad \text { and } \quad h: \mathcal{S}_{2,0} \rightarrow \mathcal{S}_{0,6}
$$

be the orbifold covering maps shown at the top left and right of Figure 5 , respectively. Given $g \geq 2$, let $\sigma_{g}: \mathcal{S}_{g, 0} \rightarrow \mathcal{S}_{2,0}$ be the covering map given at the bottom of Figure 5. Let $F_{g}=\sigma_{g} \circ h \circ f$. Let $\nu$ be the lamination $\nu=F_{g}^{-1}(\hat{\nu})$.

Recall the sequence of curves $\left\{\hat{\gamma}_{i}\right\}_{i=0}^{\infty}$. Denote the surface $S_{g, 0}$ by $S$ and the covering map $F_{g}$ by $F$.

Theorem 3.6. There are constants $k \geq 1$ and $c \geq 0$ so that the sequence $\left\{F^{-1}\left(\hat{\gamma}_{i}\right)\right\}_{i=0}^{\infty}$ is a $(k, c)$-quasi-geodesic in $\mathcal{C}(S)$. 
Proof. By Theorem 8.1 of [RS09] we have that the set-valued map that assigns to each simple closed curve $\alpha$ on the orbifold $\mathcal{S}_{0,5}$ the component curves of $F^{-1}(\alpha)$ in the orbifold cover $\mathcal{S}_{g, 0}$ is a $(Q, Q)$-quasi-isometry from $\mathcal{C}\left(S_{0,5}\right)$ to $\mathcal{C}\left(S_{g, 0}\right)$, where $Q \geq 1$ is a constant depending only on the degree of the cover $4(g-1)$. Then the theorem follows from Proposition 3.1 (3).

For each $i \geq 0$ let $\gamma_{i}$ be a component curve of $F^{-1}\left(\hat{\gamma}_{i}\right)$. By Theorem 3.6 we have

$$
d_{S}\left(\gamma_{i}, \gamma_{j}\right) \geq \frac{1}{k}|i-j|-c .
$$

Let $d=2 k+k c$. If $|i-j| \geq d$, then by the above inequality we have

$$
d_{S}\left(\gamma_{i}, \gamma_{j}\right) \geq 2
$$

which implies that

$$
\gamma_{j} \pitchfork \gamma_{i}
$$

holds.

Theorem 3.7. Let the sequence of curves $\left\{\gamma_{i}\right\}_{i=0}^{\infty}$, the marking $\mu$ and the lamination $\nu$ be as above. There are constants $K \geq 1$ and $C \geq 0$ depending only on the degree of the cover such that we have

(1) For any $i \geq d, j \geq i+d$ and $j^{\prime} \leq i-d$ we have $d_{\gamma_{i}}\left(\gamma_{j}, \gamma_{j^{\prime}}\right) \asymp_{K, C} e_{i-1}$.

(2) $d_{\gamma_{i}}(\mu, \nu) \asymp_{K, C} e_{i-1}$ for all $i \geq 1$.

(3) For any essential, non-annular subsurface $W$ we have $d_{W}(\mu, \nu) \leq R$.

(4) The lamination $\nu$ is a minimal filling non-uniquely ergodic lamination on $S_{g, 0}$.

Proof. First we prove part (4). Since $\hat{\nu}$ is a non-uniquely ergodic lamination there are curves $\alpha, \beta \in \mathcal{C}_{0}\left(S_{0,5}\right)$ and measures $\hat{m}$ and $\hat{m}^{\prime}$ supported on $\hat{\nu}$ so that

$$
\frac{\hat{m}(\alpha)}{\hat{m}^{\prime}(\alpha)} \neq \frac{\hat{m}(\beta)}{\hat{m}^{\prime}(\beta)} .
$$

Let $m=F^{*}(\hat{m})$ and $m^{\prime}=F^{*}(\hat{m})$ be the pull-backs of $\hat{m}$ and $\hat{m}^{\prime}$, respectively. Then $m$ and $m^{\prime}$ are measures supported on $\nu$. Let $\tilde{\alpha}$ be a component of $F^{-1}(\alpha)$ and $\tilde{\beta}$ be a component of $F^{-1}(\beta)$. Then $m(\tilde{\alpha})=\hat{m}(\alpha)$ and $m(\tilde{\beta})=$ $\hat{m}(\beta)$. Therefore

$$
\frac{m(\tilde{\alpha})}{m^{\prime}(\tilde{\alpha})} \neq \frac{m(\tilde{\beta})}{m^{\prime}(\tilde{\beta})},
$$

and hence the lamination $\nu$ is a non-uniquely ergodic lamination.

We proceed to show that the lamination $\nu$ is minimal and filling. We use the facts stated in $\$ 2.1$ about measured laminations and foliations and the correspondence between them. Equip $\hat{\nu}$ with a transverse measure $\hat{m}$ and $\nu$ with measure the measure $m=F^{*}(\hat{m})$. Let $(\hat{\mathcal{F}}, \hat{m})$ and $(\mathcal{F}, m)$ be the measured foliations corresponding to $(\hat{\nu}, \hat{m})$ and $(\nu, m)$, respectively. Note that $\mathcal{F}=F^{-1}(\hat{\mathcal{F}})$. Since the lamination $\hat{\nu}$ is minimal, the foliation $\hat{\mathcal{F}}$ is minimal. By the result of Hubbard and Masur HM79 given a complex structure on the surface $S_{0,5}$ there is a unique quadratic differential $\hat{q}$ with 
vertical measured foliation $(\hat{\mathcal{F}}, \hat{m})$. Then $(\mathcal{F}, m)$ is the vertical measured foliation of the quadratic differential $q=F^{*}(\hat{q})$. Since $\mathcal{F}$ is a minimal foliation on $\mathcal{S}_{0,5}$ any leaf of $\mathcal{F}$ is dense in the surface. Therefore, the lift of each leaf of $\mathcal{F}$ to $\mathcal{S}_{g, 0}$ is dense. To see this, let $l$ be a leaf of $\mathcal{F}$. Suppose to the contrary that $l$ misses an open set $U$ in $\mathcal{S}_{g, 0}$. We may shrink $U$ and assume that the restriction of $F$ to $U$ is a homeomorphism. But then $F(l)$ which is a leaf of $\hat{\mathcal{F}}$ misses $F(U)$, which is an open subset of $\mathcal{S}_{0,5}$. This contradicts the fact that $\hat{\mathcal{F}}$ is a minimal foliation of $S_{0,5}$. Therefore $\mathcal{F}$ is minimal and consequently $\nu$ is as well.

To see that the lamination $\nu$ fills $S$, note that given $\alpha \in \mathcal{C}_{0}(S)$, a homotopy that realizes $\alpha$ and $\nu$ as disjoint subsets of $\mathcal{S}_{g, 0}$ composed with $F$ gives us a homotopy which realizes $F(\alpha)$ (an essential closed curve on $S_{0,5}$ ) and $\hat{\nu}$ as disjoint subsets of $S_{0,5}$. But this contradicts the fact that $\hat{\nu}$ fills $S_{0,5}$.

Using the terminology of [RS09] we say that a subsurface $W \subseteq S_{g, 0}$ is a symmetric subsurface if it is a component of $F^{-1}(Y)$ for some subsurface $Y \subseteq S_{5,0}$.

When the subsurface $W$ is not symmetric by Lemma 7.2 of [RS09], we have

$$
d_{W}(\mu, \nu) \leq 2 T_{e}+1
$$

for a constant $T_{e}>0$ depending only on the degree of the cover and the constant $e$ which comes from Rafi's characterization of short curves along Teichmüller geodesics; see $\S 4$ of [RS09] and for more detail [Raf05], Raf14].

When the subsurface $W$ is an essential symmetric subsurface we have

$$
d_{W}(\mu, \nu) \leq d_{Y}(\hat{\mu}, \hat{\nu})
$$

(see the proof of Theorem 8.1 in [RS09]). Furthermore, by Theorem 3.4, we know that there exists $\hat{R}>0$ so that

$$
d_{Y}(\hat{\mu}, \hat{\nu}) \leq \hat{R}
$$

for every essential, non-annular subsurface $Y \subseteq S_{0,5}$. The above two inequalities for subsurface coefficients give us

$$
d_{W}(\mu, \nu) \leq \hat{R}
$$

Then by the subsurface coefficient bounds (3.17) and (3.18) we obtain the upper bound $R:=\max \left\{\hat{R}, 2 T_{e}+1\right\}$ in part (3).

We proceed to prove parts (1) and (2). The fact that the subsurface coefficient $d_{\gamma_{i}}\left(\gamma_{j}, \gamma_{j^{\prime}}\right)$ in part (1) is defined follows from (3.16). Note that each annular subsurface with core curve $\gamma_{i}$ is a symmetric subsurface, because $\gamma_{i}$ is a component of $F^{-1}\left(\hat{\gamma}_{i}\right)$. Thus as is shown in the proof of Theorem 8.1 in [RS09] there exists $Q \geq 1$ so that

$$
\begin{array}{rll}
d_{\gamma_{i}}\left(\gamma_{j}, \gamma_{j^{\prime}}\right) & \asymp_{Q, Q} & d_{\hat{\gamma}_{i}}\left(\hat{\gamma}_{j}, \hat{\gamma}_{j^{\prime}}\right), \text { and } \\
d_{\gamma_{i}}(\mu, \nu) & \asymp_{Q, Q} & d_{\hat{\gamma}_{i}}(\hat{\nu}, \hat{\mu}) .
\end{array}
$$



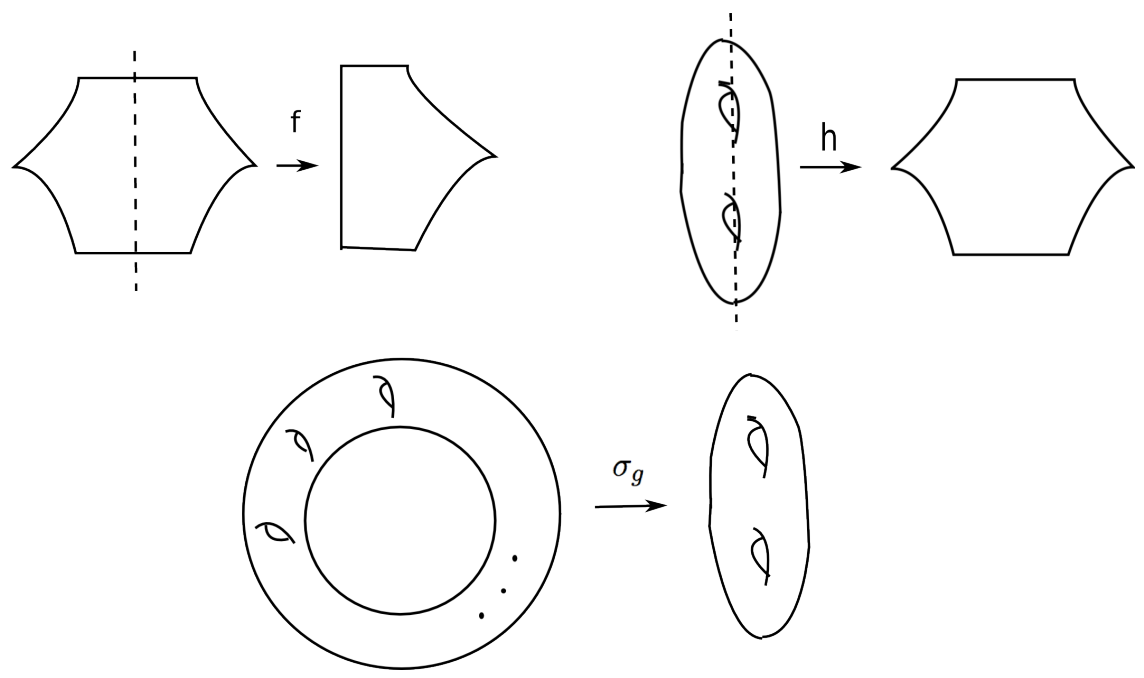

Figure 5. Top left: $f: \mathcal{S}_{0,6} \rightarrow \mathcal{S}_{0,5}$ is the orbifold covering map realized as the rotation by angle $\pi$ about the axis in the picture. Top right: $h: \mathcal{S}_{2,0} \rightarrow \mathcal{S}_{0,6}$ is the orbifold covering map realized as the rotation by angle $\pi$ about the axis in the picture (hyperelliptic involution). Bottom: $\sigma_{g}: \mathcal{S}_{g, 0} \rightarrow \mathcal{S}_{2,0}$ is the covering map realized by $g-1$ times iteration of the rotation by angle $\frac{2 \pi}{g-1}$.

By Proposition 3.1 (4) we have

$$
d_{\hat{\gamma}_{i}}\left(\hat{\gamma}_{j}, \hat{\gamma}_{j^{\prime}}\right) \asymp e_{i-1},
$$

then from the quasi-equality of subsurface coefficients (3.19) the quasiequality (1) follows.

Moreover, by Proposition 3.3 (4) we have

$$
d_{\hat{\gamma}_{i}}(\hat{\mu}, \hat{\nu}) \asymp e_{i-1},
$$

then from the quasi-equality of subsurface coefficients (3.20) the quasiequality (2) follows.

\section{ReCURRENCE of GeOdesics}

Let $X \in \operatorname{Teich}(S)$ and $\nu^{-}$be a Bers marking of $X$. Let $\nu^{+}$be a minimal filling lamination. By Lemma 2.12 there is an infinite WP geodesic ray $r:[0, \infty) \rightarrow \operatorname{Teich}(S)$ with $r(0)=X$ and end invariant $\left(\nu^{-}, \nu^{+}\right)$. Denote the projection of $r$ to the moduli space by $\hat{r}$. For $\epsilon>0$, the $\epsilon$-thick part of $\mathcal{M}(S)$ consists of the Riemann surfaces with injectivity radius greater than $\epsilon$.

Theorem 4.1. Given $R>0$. Suppose that the pair $\left(\nu^{-}, \nu^{+}\right)$has nonannular $R$-bounded combinatorics. There is an $\epsilon>0$ such that $\hat{r}$ is recurrent to the $\epsilon$-thick part of the moduli space. 
Lemma 4.2. Given $R>0$, there are constants $T_{0}>0$ and $\epsilon>0$ with the following property. Let $T>T_{0}$ and $\zeta_{n}:[0, T] \rightarrow \operatorname{Teich}(S)$ be a sequence of WP geodesic segments parametrized by arc-length so that the pair $\left(Q\left(\zeta_{0}(0)\right), Q\left(\zeta_{n}(T)\right)\right)$ has non-annular $R$-bounded combinatorics. Then there is a time $t^{*} \in[0, T]$ and a sequence $\left\{m_{n}\right\}_{n=1}^{\infty}$ so that for all $n$ sufficiently large we have

$$
\operatorname{inj}\left(\zeta_{m_{n}}\left(t^{*}\right)\right)>\epsilon
$$

Proof. Consider the limiting picture of geodesic segments $\zeta_{n}$ as was described in Theorem 2.13. Let the partition $0=t_{0}<\ldots<t_{k+1}=T$, the multi-curves $\sigma_{i}, i=0, \ldots, k+1$, the multi-curve $\hat{\tau}$, and the piecewise geodesic

$$
\hat{\zeta}:[0, T] \rightarrow \overline{\operatorname{Teich}(S)}
$$

be as in the theorem. Furthermore, recall the elements of the mapping class group $\psi_{n}$ for $n \in \mathbb{N}$, and $\mathcal{T}_{i, n} \in \operatorname{tw}\left(\sigma_{i}-\hat{\tau}\right)$ for $i=0, \ldots, k+1$ and $n \in \mathbb{N}$. Also as in the theorem set $\varphi_{i, n}=\mathcal{T}_{i, n} \circ \ldots \circ \mathcal{T}_{1, n} \circ \psi_{n}$.

First we show that $\hat{\tau}=\emptyset$. Suppose to the contrary that $\hat{\tau} \neq \emptyset$.

From Theorem 2.13 we know that $\hat{\tau}=\sigma_{0} \cap \sigma_{1}$, so $\hat{\tau} \subseteq \sigma_{0}$. Moreover by Theorem $2.13(2), \hat{\zeta}(0) \in \mathcal{S}\left(\sigma_{0}\right)$. Thus for any $\alpha \in \hat{\tau}$ we have $\ell_{\alpha}(\hat{\zeta}(0))=0$. Furthermore, by Theorem 2.13 (3), after possibly passing to a subsequence $\psi_{n}\left(\zeta_{n}(0)\right) \rightarrow \hat{\zeta}(0)$ as $n \rightarrow \infty$. Thus by continuity of length functions for all $n$ sufficiently large and any $\alpha \in \hat{\tau}, \ell_{\alpha}\left(\psi_{n}\left(\zeta_{n}(0)\right) \leq L_{S}\right.$. Thus there is $Q_{0, n}$ a Bers pants decomposition of $\psi_{n}\left(\zeta_{n}(0)\right)$ that contains $\hat{\tau}$.

Similarly since $\hat{\tau}=\sigma_{k} \cap \sigma_{k+1}$ (as in Theorem 2.13), we have $\hat{\tau} \subseteq \sigma_{k+1}$. Moreover, by Theorem 2.13 2), we know that $\hat{\zeta}(T) \in \mathcal{S}\left(\sigma_{k+1}\right)$. Thus for any $\alpha \in \hat{\tau}$, we have $\ell_{\alpha}(\hat{\zeta(T)})=0$. Furthermore, by Theorem 2.13 (3) after possibly passing to a subsequence, $\varphi_{k, n}\left(\zeta_{n}(T)\right) \rightarrow \hat{\zeta}(T)$ as $n \rightarrow \infty$. Thus by continuity of length functions for all $n$ sufficiently large and any $\alpha \in \hat{\tau}$, we have that $\ell_{\alpha}\left(\varphi_{k, n}\left(\zeta_{n}(T)\right) \leq L_{S}\right.$.

Now note that we have $\varphi_{k, n}=\mathcal{T}_{k, n} \circ \ldots \circ \mathcal{T}_{1, n} \circ \psi_{n}$. The element of mapping class group $\mathcal{T}_{i, n}$ is a composition of powers of Dehn twists about the curves in $\sigma_{i}$ and $\hat{\tau} \subseteq \sigma_{i}$. Therefore, $\mathcal{T}_{i, n}$ preserves the isotopy class and the length of every curve $\alpha \in \hat{\tau}$. Thus applying $\left(\mathcal{T}_{k, n} \circ \ldots \circ \mathcal{T}_{1, n}\right)^{-1}$ to $\ell_{\alpha}\left(\varphi_{k, n}\left(\zeta_{n}(T)\right)\right)$, we obtain

$$
\ell_{\alpha}\left(\varphi_{k, n}\left(\zeta_{n}(T)\right)\right)=\ell_{\alpha}\left(\psi_{n}\left(\zeta_{n}(T)\right)\right) .
$$

Then by the previous paragraph for all $n$ sufficiently large, $\ell_{\alpha}\left(\psi_{n}\left(\zeta_{n}(T)\right)\right) \leq$ $L_{S}$. Thus there is a Bers pants decomposition $Q_{k+1, n}$ of $\psi_{n}\left(\zeta_{n}(T)\right)$ containing $\hat{\tau}$.

Let the threshold constant in the distance formula (2.4) be $A>\max \left\{M_{1}, R, 2\right\}$. Then there are constants $K \geq 1$ and $C \geq 0$ such that

$$
d\left(Q_{0, n}, Q_{k+1, n}\right) \asymp_{K, C} \sum_{\substack{Y \subseteq S \\ \text { non-annular }}}\left\{d_{Y}\left(Q_{0, n}, Q_{k+1, n}\right)\right\}_{A} .
$$


As we saw above $\hat{\tau} \subset Q_{0, n}$ and $\hat{\tau} \subset Q_{k+1, n}$. So for any essential subsurface $W$ satisfying $\hat{\tau} \pitchfork W$, it follows that

$$
d_{W}\left(Q_{0, n}, Q_{k+1, n}\right) \leq 2 .
$$

Thus subsurfaces which overlap $\hat{\tau}$ do not contribute to the right hand side of (4.1). On the other hand, by Theorem 2.11 (Quasi-Isometric Model) there are constants $K_{\mathrm{WP}} \geq 1, C_{\mathrm{WP}} \geq 0$ such that

$$
d\left(Q_{0, n}, Q_{k+1, n}\right) \asymp_{K_{\mathrm{WP}}, C_{\mathrm{WP}}} d_{\mathrm{WP}}\left(\zeta_{n}(0), \zeta_{n}(T)\right) .
$$

Let $T_{0}=K_{\mathrm{WP}}(K A+K C)+K_{\mathrm{WP}} C_{\mathrm{WP}}$. Since $T \geq T_{0}$ by the above quasiequality

$$
d\left(Q_{0, n}, Q_{k+1, n}\right) \geq K A+K C .
$$

Now (4.1) and the above inequality imply that for any $n \in \mathbb{N}$, there is an essential non-annular subsurface $Y_{n}$ with

$$
d_{Y_{n}}\left(Q_{0, n}, Q_{k+1, n}\right) \geq A \geq R .
$$

But as we saw above $Y_{n}$ can not overlap $\hat{\tau}$ (otherwise $d_{Y_{n}}\left(Q_{0, n}, Q_{k+1, n}\right) \leq$ $2<A$ ), therefore $Y_{n} \subseteq S \backslash \hat{\tau}$. Moreover, since $\hat{\tau} \neq \emptyset, Y_{n}$ is a proper subsurface. Applying $\psi_{n}^{-1}$ to the subsurface coefficient above we get

$$
d_{\psi_{n}^{-1}\left(Y_{n}\right)}\left(\psi_{n}^{-1}\left(Q_{0, n}\right), \psi_{n}^{-1}\left(Q_{k+1, n}\right)\right) \geq R,
$$

where $\psi_{n}^{-1}\left(Q_{0, n}\right)$ is a Bers pants decomposition of $\zeta_{n}(0)$ and $\psi_{n}^{-1}\left(Q_{k+1, n}\right)$ is a Bers pants decomposition of $\zeta_{n}(T)$. Moreover, $\psi_{n}^{-1}\left(Y_{n}\right)$ is a proper subsurface of $S$, because $Y_{n}$ is a proper subsurface of $S$. But then the lower bound 4.2 contradicts the non-annular bounded combinatorics assumption for the two pants decompositions $Q\left(\zeta_{n}(0)\right)$ and $Q\left(\zeta_{n}(T)\right)$. This contradiction completes the proof of the fact that $\hat{\tau}=\emptyset$.

Let $t^{*}=\frac{t_{1}}{2}$. By Theorem 2.13 (2) and since $\hat{\tau}=\emptyset$, we have that $\hat{\zeta}\left(t^{*}\right) \in$ $\operatorname{Teich}(S)$. So $\operatorname{inj}\left(\hat{\zeta}\left(t^{*}\right)\right)>2 \epsilon$ for some $\epsilon>0$. Furthermore, by Theorem 2.13 (3), there is a sequence $\left\{m_{n}\right\}_{n=1}^{\infty}$ such that $\psi_{m_{n}}\left(\zeta_{m_{n}}\left(t^{*}\right)\right) \rightarrow \hat{\zeta}\left(t^{*}\right)$ as $n \rightarrow \infty$. Therefore, $\operatorname{inj}\left(\psi_{m_{n}}\left(\zeta_{m_{n}}\left(t^{*}\right)\right)\right)>\epsilon$ for any $n$ sufficiently large. Then since the action by elements of the mapping class group does not change the injectivity radius of a surface $\operatorname{inj}\left(\zeta_{m_{n}}\left(t^{*}\right)\right)>\epsilon$. The lemma is proved.

Proof of Theorem 4.1. Let $T_{0}>0$ be as in Lemma 4.2 and $T \geq T_{0}$. Consider the sequence of WP geodesic segments

$$
\zeta_{n}:=\left.r\right|_{[n T,(n+1) T]}:[0, T] \rightarrow \operatorname{Teich}(S) .
$$

Note that Theorem 2.10 guarantees that for $D=d_{R}\left(K_{\mathrm{WP}}, C_{\mathrm{WP}}\right)$, the paths $Q(r)$ and $\rho, D$-fellow travel in the pants graph. Let $z_{n}^{-}, z_{n}^{+} \in[0, \infty)$ be so that

$$
\begin{aligned}
d\left(\rho\left(z_{n}^{-}\right), Q\left(\zeta_{n}(0)\right)\right) & \leq D, \text { and } \\
d\left(\rho\left(z_{n}^{+}\right), Q\left(\zeta_{n}(T)\right)\right) & \leq D .
\end{aligned}
$$


Then for every essential non-annular subsurface $Y \subsetneq S$,

$$
\begin{aligned}
d_{Y}\left(\rho\left(z_{n}^{-}\right), Q\left(\zeta_{n}(0)\right)\right) & \leq D, \text { and } \\
d_{Y}\left(\rho\left(z_{n}^{+}\right), Q\left(\zeta_{n}(T)\right)\right) & \leq D .
\end{aligned}
$$

Moreover by the assumption that the pair $\left(\nu^{-}, \nu^{+}\right)$has non-annular $R$-bounded combinatorics for any proper, essential non-annular subsurfaces $Y \subsetneq S$ we have, $d_{Y}\left(\nu^{-}, \nu^{+}\right) \leq R$. Then by the no back tracking property of Hierarchy paths (Theorem 2.8) there is an $M_{2}>0$ so that

$$
d_{Y}\left(\rho\left(z_{n}^{-}\right), \rho\left(z_{n}^{+}\right)\right) \leq R+2 M_{2} .
$$

The subsurface coefficient bounds (4.3), (4.4) and 4.5) combined with the triangle inequality imply that

$$
\begin{aligned}
d_{Y}\left(Q\left(\zeta_{n}(0)\right), Q\left(\zeta_{n}(T)\right)\right) & \leq 2 D+R+2 M_{2}+\operatorname{diam}_{Y}\left(\rho\left(z_{n}^{-}\right)\right)+\operatorname{diam}_{Y}\left(\rho\left(z_{n}^{+}\right)\right) \\
& \leq 2 D+R+2 M_{2}+4 .
\end{aligned}
$$

Thus the pair $\left(Q\left(\zeta_{n}(0)\right), Q\left(\zeta_{n}(T)\right)\right)$ has $R+2 D+2 M_{2}+4$ non-annular bounded combinatorics.

Then Lemma 4.2 applies to the sequence of geodesic segments $\zeta_{n}:=$ $\left.r\right|_{[n T,(n+1) T]}$ and implies that there are $t^{*} \in[0, T], \epsilon>0$ and a sequence of integers $\left\{m_{n}\right\}_{n=1}^{\infty}$ such that at $a_{n}=m_{n} T+t^{*}$ we have

$$
\operatorname{inj}\left(r\left(a_{n}\right)\right)>\epsilon \text {. }
$$

This implies that $\hat{r}\left(a_{n}\right)$ where $\hat{r}$ is the projection of $r$ to the moduli space is in the $\epsilon$-thick part of the moduli space. Furthermore, since $a_{n} \rightarrow \infty$, the ray is recurrent to the $\epsilon$-thick part of the moduli space.

Let $r:[0, \infty) \rightarrow$ Teich $(S)$ be the ray with end invariant $\left(\nu^{-}, \nu^{+}\right)$with non-annular bounded combinatorics. In Theorem 4.1 we saw that the ray $\hat{r}$ is recurrent to a compact subset of $\mathcal{M}(S)$. In Theorem 4.4 we show that if in addition there is a sequence of curves $\left\{\gamma_{i}\right\}_{i=1}^{\infty}$ so that $d_{\gamma_{i}}\left(\nu^{-}, \nu^{+}\right) \rightarrow \infty$ as $i \rightarrow \infty$, then the recurrent ray $\hat{r}$ is not contained in any compact part of the moduli space. The theorem also follows from Theorem 3.1 of [BMM11]. The proof here is different and more direct and gives some information about the excursion times. We need the following result from $\S 4$ of [Mod].

Lemma 4.3. (Large twist $\Longrightarrow$ Short curve) Given $T, \epsilon_{0}$ and $N$ positive, there is an $\epsilon<\epsilon_{0}$ with the following property. Let $\zeta:\left[0, T^{\prime}\right] \rightarrow \operatorname{Teich}(S)$ be a WP geodesic segment of length $T^{\prime} \leq T$ such that

$$
\sup _{t \in\left[0, T^{\prime}\right]} \ell_{\gamma}(\zeta(t)) \geq \epsilon_{0}
$$

If $d_{\gamma}\left(\mu(\zeta(0)), \mu\left(\zeta\left(T^{\prime}\right)\right)\right)>N(\mu(X)$ denotes a Bers marking of the point $X \in \operatorname{Teich}(S))$, then we have

$$
\inf _{t \in\left[0, T^{\prime}\right]} \ell_{\gamma}(\zeta(t)) \leq \epsilon .
$$

Moreover, $\epsilon \rightarrow 0$ as $N \rightarrow \infty$. 
Theorem 4.4. Let $r:[0, \infty) \rightarrow$ Teich $(S)$ be a WP geodesic ray with end invariant $\left(\nu^{-}, \nu^{+}\right)$. Suppose $\left(\nu^{-}, \nu^{+}\right)$has non-annular $R$-bounded combinatorics. Moreover assume that there is a sequence of curves $\left\{\gamma_{i}\right\}_{i=1}^{\infty}$ so that

$$
d_{\gamma_{i}}\left(\nu^{-}, \nu^{+}\right) \rightarrow \infty
$$

as $i \rightarrow \infty$. Then there is a sequence of times $b_{i} \rightarrow \infty$ as $i \rightarrow \infty$ such that

$$
\ell_{\gamma_{i}}\left(r\left(b_{i}\right)\right) \rightarrow 0
$$

as $i \rightarrow \infty$.

Proof. Let $\rho:[0, \infty) \rightarrow P(S)$ be a hierarchy path with end points $\nu^{-}$and $\nu^{+}$. The pair $\left(\nu^{-}, \nu^{+}\right)$has non-annular bounded combinatorics, so Theorem 2.10 implies that for $D=d_{R}\left(K_{\mathrm{WP}}, C_{\mathrm{WP}}\right), \rho$ and $Q(r), D$-fellow travel. Moreover both $\rho$ and $Q(r)$ are quasi-geodesics. Thus there is a quasi-isometry $N$ : $[0, \infty] \rightarrow[0, \infty)$ from the domain of $\rho$ to the domain of $r$. The map assigns to each $i$ in the domain of $\rho$ any time $t$ in the smallest interval in the domain of $r$ which contains all $t^{\prime}$ with $d\left(\rho(i), Q\left(r\left(t^{\prime}\right)\right)\right) \leq D$. For more detail see $\S 5$ of [Mod]. Denote the constants of the quasi-isometry $N$ by $K_{1}$ and $C_{1}$.

We assumed that $d_{\gamma_{i}}\left(\nu^{-}, \nu^{+}\right) \rightarrow \infty$ as $i \rightarrow \infty$ so for all $i$ sufficiently large

$$
d_{\gamma_{i}}\left(\nu^{-}, \nu^{+}\right) \geq M_{1}
$$

where $M_{1}$ is the constant from the Large Link Lemma ([MM00, Lemma 6.2]), see also property (2) of hierarchy paths in [BMM11, Theorem 2.6]. Then the annular subsurface with core curve $\gamma_{i}$ is a component domain of $\rho$. Thus there is a time $q_{i} \in[0, \infty]$, so that $\rho\left(q_{i}\right)$ contains the curve $\gamma_{i}$. Note that the sequence of times $q_{i} \rightarrow \infty$ as $i \rightarrow \infty$.

Since the pair $\left(\nu^{-}, \nu^{+}\right)$has non-annular $R$-bounded combinatorics, for any proper, essential non-annular subsurfaces $Y \subsetneq S$ we have $d_{Y}\left(\nu^{-}, \nu^{+}\right) \leq$ $R$. Then by the no back tracking property of hierarchy paths (Theorem 2.8) there is an $M_{2}>0$ so that for any $i, j \in \mathbb{N}$ we have

$$
d_{Y}\left(\rho\left(q_{i}\right), \rho\left(q_{j}\right)\right) \leq R+2 M_{2} .
$$

Let the threshold in the distance formula (2.4) be $\max \left\{M_{1}, R+2 M_{2}\right\}$. Then there are constants $K_{R} \geq 1$ and $C_{R} \geq 0$ corresponding to the threshold so that

$$
d\left(\rho\left(q_{i}\right), \rho\left(q_{j}\right)\right) \asymp_{K_{R}, C_{R}} d_{S}\left(\rho\left(q_{i}\right), \rho\left(q_{j}\right)\right) .
$$

Let $\mathbf{w}(D, 0, R)$ be the constant from the Annular Coefficient Comparison Lemma in $\S 6$ of [Mod], see below. Let $w=\max \{\mathbf{w}, 2\}$. We have that the pair $\left(\nu^{-}, \nu^{+}\right)$has non-annular $R$-bounded combinatorics. Moreover for each $i$ sufficiently large $\gamma_{i} \in \rho\left(q_{i}\right)$. These two facts imply that for all $i$ sufficiently large, the curve $\gamma_{i}$ is $(w, 0)$-isolated at $q_{i}$, where the subsurface with nonannular $R$-bounded combinatorics on both sides of $q_{i}$ is the surface $S$. See [Mod, §6.1] for the definition of isolated curve (isolated annular subsurface) along a hierarchy path. 
Recall that $\rho$ is a $(k, c)$-quasi-geodesic in $P(S)$. Let $K_{2}=\max \left\{K_{1}, K_{R}\right\}$ and $C_{2}=\max \left\{C_{1}, C_{R}\right\}$, where $K_{1}, C_{1}$ are the constants of the quasi-isometry $N$ and $K_{R}, C_{R}$ are the constants in the quasi-equality (4.6). For any integer $i \geq 0$, set

$$
q_{i}^{-}=q_{i}-k\left(K_{2}\left(w+C_{2}\right)\right)-k c \text { and } q_{i}^{+}=q_{i}+k\left(K_{2}\left(w+C_{2}\right)\right)+k c .
$$

By the setup of $q_{i}^{-}$for any $q \leq q_{i}^{-}$we have $d\left(\rho\left(q_{i}\right), \rho(q)\right) \geq K_{2}\left(w+C_{2}\right)$, and by the setup of $q_{i}^{+}$for any $q \geq q_{i}^{+}, d\left(\rho\left(q_{i}\right), \rho(q)\right) \geq K_{2}\left(w+C_{2}\right)$. Then the quasi-equality (4.6) implies that

$$
d_{S}\left(\rho\left(q_{i}\right), \rho(q)\right) \geq w \geq 2 .
$$

The above inequality guarantees that the $\mathcal{C}(S)$-distance of any curve in in the pants decomposition $\rho\left(q_{i}\right)$ and any curve in in the pants decomposition $\rho(q)$ is at least 2 . Thus any curve $\rho\left(q_{i}\right)$ intersects any curve in $\rho(q)$. In particular, $\gamma_{i}$ intersects any curve in $\rho(q)$. Then there is an $M_{3}>0$, so that $d_{\gamma_{i}}\left(\rho\left(q_{i}^{+}\right), \nu^{+}\right) \leq M_{3}$ and $d_{\gamma_{i}}\left(\rho\left(q_{i}^{-}\right), \nu^{-}\right) \leq M_{3}$, see property (4) of hierarchy paths in [BMM11, Theorem 2.6]. Therefore,

$$
d_{\gamma_{i}}\left(\rho\left(q_{i}^{-}\right), \rho\left(q_{i}^{+}\right)\right) \asymp_{1,2 M_{3}} d_{\gamma_{i}}\left(\nu^{-}, \nu^{+}\right) .
$$

Let $s_{i}^{-} \in N\left(\rho\left(q_{i}^{-}\right)\right)$and $s_{i}^{+} \in N\left(\rho\left(q_{i}^{+}\right)\right)$. Since $q_{i}^{+}-q_{i} \geq \mathbf{w}$ and $q_{i}-q_{i}^{-} \geq \mathbf{w}$ by Annular Coefficient Comparison Lemma in [Mod, §6] we have

- $\min \left\{\ell_{\gamma_{i}}\left(r\left(s_{i}^{-}\right)\right), \ell_{\gamma_{i}}\left(r\left(s_{i}^{+}\right)\right)\right\} \geq \omega\left(L_{S}\right)$, where $\omega(a)$ is the width of the collar of a simple closed geodesic with length $a$ on a complete hyperbolic surface provided by the Collar Lemma (see [Bus10, §4.1]), and

- $d_{\gamma_{i}}\left(Q\left(r\left(s_{i}^{-}\right)\right), Q\left(r\left(s_{i}^{+}\right)\right)\right) \asymp_{1, B} d_{\gamma_{i}}\left(\rho\left(q_{i}^{-}\right), \rho\left(q_{i}^{+}\right)\right)$, for a constant $B$ depending only on $D$.

By the setup of $q_{i}^{-}$and $q_{i}^{+}$we have $q_{i}^{+}-q_{i}^{-} \leq L$, where

$$
L=2 k\left(K_{2}\left(w+C_{2}\right)\right)+2 k c .
$$

Then since $N$ is a $\left(K_{1}, C_{1}\right)$-quasi-isometry the length of the interval $\left[s_{i}^{-}, s_{i}^{+}\right]$ is bounded above by $K_{1} L+C_{1}$. This fact and the first bullet above allow us to apply Lemma 4.3 to the geodesic segment $\left.r\right|_{\left[s_{i}^{-}, s_{i}^{+}\right]}$and conclude that there exists $\epsilon_{i}>0$ depending on the upper bound for the length of the interval $\left[s_{i}^{-}, s_{i}^{+}\right]$, the lower bound $\omega\left(L_{S}\right)$ in the first bullet above and the value of the annular coefficient $d_{\gamma_{i}}\left(Q\left(r\left(s_{i}^{-}\right)\right), Q\left(r\left(s_{i}^{+}\right)\right)\right)$so that

$$
\inf _{t \in\left[s_{i}^{-}, s_{i}^{+}\right]} \ell_{\gamma_{i}}(r(t)) \leq \epsilon_{i} .
$$

Moreover, the second bullet above, the quasi-equality (4.7) and the assumption that

$$
d_{\gamma_{i}}\left(\nu^{-}, \nu^{+}\right) \rightarrow \infty
$$

as $i \rightarrow \infty$, together imply that

$$
d_{\gamma_{i}}\left(Q\left(r\left(s_{i}^{-}\right)\right), Q\left(r\left(s_{i}^{+}\right)\right)\right) \rightarrow \infty .
$$


as $i \rightarrow \infty$. Then the last statement of Lemma 4.3 guarantees that $\epsilon_{i} \rightarrow 0$ as $i \rightarrow \infty$.

Let $b_{i} \in\left[s_{i}^{-}, s_{i}^{+}\right]$be the time that the above infimum is realized. Then $\ell_{\gamma_{i}}\left(r\left(b_{i}\right)\right) \rightarrow 0$ as $i \rightarrow \infty$. Moreover since $q_{i} \rightarrow \infty$ as $i \rightarrow \infty$, we have $q_{i}^{-} \rightarrow \infty$ as $i \rightarrow \infty$. Thus $s_{i}^{-} \rightarrow \infty$ as $i \rightarrow \infty$. Then $b_{i} \rightarrow \infty$ as $i \rightarrow \infty$. This completes the proof of the lemma.

Proof of Theorem 1.1. Let $\left\{\gamma_{i}\right\}_{i=0}^{\infty}$ be a sequence of curves as in $\oint 3$ and let $\nu^{+}$be the minimal filling non-uniquely ergodic lamination in $\mathcal{E} \mathcal{L}(S)$ which is determined by the sequence. Let $\nu^{-}$be a marking containing $\gamma_{0}, \ldots, \gamma_{3}$ as in $\$ 3$. Then by Theorem 3.7 (3), the pair $\left(\nu^{-}, \nu^{+}\right)$has non-annular $R$-bounded combinatorics. Let $X \in \operatorname{Teich}(S)$ be a point with a Bers marking $\nu^{-}$. By Lemma 2.12 there is a geodesic ray $r:[0, \infty) \rightarrow \operatorname{Teich}(S)$ with $r(0)=X$ and the forward ending lamination $\nu^{+}$. Then Theorem 4.1 implies that $\hat{r}$ is recurrent to a compact subset of $\mathcal{M}(S)$. Furthermore, by Theorem 3.7 (2),

$$
d_{\gamma_{i}}\left(\nu^{-}, \nu^{+}\right) \geq \frac{1}{K} e_{i-1}-C .
$$

Then since $e_{i} \rightarrow \infty$ as $i \rightarrow \infty$, we have $d_{\gamma_{i}}\left(\nu^{-}, \nu^{+}\right) \rightarrow \infty$ as $i \rightarrow \infty$. Thus by Theorem 4.4 the ray $\hat{r}$ is not contained in any compact subset of $\mathcal{M}(S)$.

Remark 4.5. Masur's criterion (Theorem 1.2) guarantees that any Teichmüller geodesic ray with vertical lamination $\nu^{+}$is divergent in $\mathcal{M}(S)$.

\section{REFERENCES}

[Aou13] Tarik Aougab, Uniform hyperbolicity of the graphs of curves, Geom. Topol. 17 (2013), no. 5, 2855-2875.

[Beh06] Jason A. Behrstock, Asymptotic geometry of the mapping class group and Teichmüller space, Geom. Topol. 10 (2006), 1523-1578.

[BH99] Martin R. Bridson and André Haefliger, Metric spaces of non-positive curvature, Grundlehren der Mathematischen Wissenschaften, vol. 319, Springer-Verlag, Berlin, 1999.

[BM08] Jeffrey Brock and Howard Masur, Coarse and synthetic Weil-Petersson geometry: quasi-flats, geodesics and relative hyperbolicity, Geom. Topol. 12 (2008), no. 4, 2453-2495.

[BMM10] Jeffrey Brock, Howard Masur, and Yair Minsky, Asymptotics of Weil-Petersson geodesics. I. Ending laminations, recurrence, and flows, Geom. Funct. Anal. 19 (2010), no. 5, 1229-1257.

[BMM11] _ Asymptotics of Weil-Petersson geodesics II: bounded geometry and unbounded entropy, Geom. Funct. Anal. 21 (2011), no. 4, 820-850.

[Bon01] Francis Bonahon, Geodesic laminations on surfaces, Laminations and foliations in dynamics, geometry and topology (Stony Brook, NY, 1998), Contemp. Math., vol. 269, Amer. Math. Soc., Providence, RI, 2001, pp. 1-37.

[Bro03] Jeffrey F. Brock, The Weil-Petersson metric and volumes of 3-dimensional hyperbolic convex cores, J. Amer. Math. Soc. 16 (2003), no. 3, 495-535.

[Bus10] Peter Buser, Geometry and spectra of compact Riemann surfaces, Modern Birkhäuser Classics, Birkhäuser Boston Inc., Boston, MA, 2010, Reprint of the 1992 edition. 
[CEG06] R. D. Canary, D. B. A. Epstein, and P. L. Green, Notes on notes of Thurston [mr0903850], Fundamentals of hyperbolic geometry: selected expositions, London Math. Soc. Lecture Note Ser., vol. 328, Cambridge Univ. Press, Cambridge, 2006, With a new foreword by Canary, pp. 1-115.

[DW03] Georgios Daskalopoulos and Richard Wentworth, Classification of WeilPetersson isometries, Amer. J. Math. 125 (2003), no. 4, 941-975.

[FLM01] Benson Farb, Alexander Lubotzky, and Yair Minsky, Rank-1 phenomena for mapping class groups, Duke Math. J. 106 (2001), no. 3, 581-597.

[FLP79] A. Fathi, F. Laudenbach, and V. Poenaru, Travaux de Thurston sur les surfaces, Astérisque No. 66-67 (1979), 1-286.

[Gab09] David Gabai, Almost filling laminations and the connectivity of ending lamination space, Geom. Topol. 13 (2009), no. 2, 1017-1041.

[HM79] John Hubbard and Howard Masur, Quadratic differentials and foliations, Acta Math. 142 (1979), no. 3-4, 221-274.

[HPW15] Sebastian Hensel, Piotr Przytycki, and Richard Webb, 1-slim triangles and uniform hyperbolicity for arc graphs and curve graphs, J. Eur. Math. Soc. (JEMS) 17 (2015), no. 4, 755-762.

[Kla] Erica Klarreich, The boundary at infinity of the curve complex, Preprint, 1999.

[Lev83] Gilbert Levitt, Foliations and laminations on hyperbolic surfaces, Topology 22 (1983), no. 2, 119-135.

[LLR] Christopher Leininger, Anna Lenzhen, and Kasra Rafi, Limit sets of Teichmüller geodesics with minimal non-uniquely ergodic vertical foliation, arXiv: 1312.2305 .

[Mas76] Howard Masur, Extension of the Weil-Petersson metric to the boundary of Teichmuller space, Duke Math. J. 43 (1976), no. 3, 623-635.

[Mas92] Hausdorff dimension of the set of nonergodic foliations of a quadratic differential, Duke Math. J. 66 (1992), no. 3, 387-442.

[MM99] Howard A. Masur and Yair N. Minsky, Geometry of the complex of curves. I. Hyperbolicity, Invent. Math. 138 (1999), no. 1, 103-149.

[MM00] H. A. Masur and Y. N. Minsky, Geometry of the complex of curves. II. Hierarchical structure, Geom. Funct. Anal. 10 (2000), no. 4, 902-974.

[Mod] Babak Modami, Prescribing the behavior of Weil-Petersson geodesics in the moduli space of Riemann surfaces, J. Topol. Anal. to appear, arXiv:1212.0051.

[Raf05] Kasra Rafi, A characterization of short curves of a Teichmüller geodesic, Geom. Topol. 9 (2005), 179-202.

[Raf14] Hyperbolicity in Teichmüller space, Geom. Topol. 18 (2014), no. 5, 3025-3053.

[RS09] Kasra Rafi and Saul Schleimer, Covers and the curve complex, Geom. Topol. 13 (2009), no. 4, 2141- 2162.

[Ser85] Caroline Series, The modular surface and continued fractions, J. London Math. Soc. (2) 31 (1985), no. 1, 69-80.

[Wol03] Scott A. Wolpert, Geometry of the Weil-Petersson completion of Teichmüller space, Surveys in differential geometry, Vol. VIII (Boston, MA, 2002), Surv. Differ. Geom., VIII, Int. Press, Somerville, MA, 2003, pp. 357-393.

[Wol10] , Families of Riemann surfaces and Weil-Petersson geometry, CBMS Regional Conference Series in Mathematics, vol. 113, Published for the Conference Board of the Mathematical Sciences, Washington, DC, 2010. 
Department of Mathematics, Brown University, Providence, Ri,

E-mail address: brock@math.brown.edu

Department of Mathematics, University of Illinois at Urbana-Champaign, 1409 W GreEN ST, URBANA, IL

E-mail address: bmodami@illinois.edu 